\title{
The top-degree part in the Matchings-Jack Conjecture
}

\author{
Adam Burchardt* \\ Jagiellonian University \\ Krakow, Poland \\ adam.burchardt@amu.edu.pl
}

Submitted: Dec 4, 2019; Accepted: Mar 7, 2021; Published: May 7, 2021

(c) Adam Burchardt. Released under the CC BY-ND license (International 4.0).

\begin{abstract}
In 1996 Goulden and Jackson introduced a family of coefficients $\left(c_{\pi, \sigma}^{\lambda}\right)$ indexed by triples of partitions which arise in the power sum expansion of some Cauchy sum for Jack symmetric functions $\left(J_{\pi}^{(\alpha)}\right)$. The coefficients $c_{\pi, \sigma}^{\lambda}$ can be viewed as an interpolation between the structure constants of the class algebra and the double coset algebra. Goulden and Jackson suggested that the coefficients $c_{\pi, \sigma}^{\lambda}$ are polynomials in the variable $\beta:=\alpha-1$ with non-negative integer coefficients and that there is a combinatorics of matching hidden behind them. This Matchings-Jack Conjecture remains open. Dołęga and Féray showed the polynomiality of connection coefficients $c_{\pi, \sigma}^{\lambda}$ and gave an upper bound on the degrees. We show a dual approach to this problem and investigate Jack characters and their connection coefficients. We give a necessary and sufficient condition for the polynomial $c_{\pi, \sigma}^{\lambda}$ to achieve this bound. We show that the leading coefficient of $c_{\pi, \sigma}^{\lambda}$ is a positive integer and we present it in the context of Matchings-Jack Conjecture of Goulden and Jackson.
\end{abstract}

Mathematics Subject Classifications: 05C10, 05C30, 05E05, 20C30

\section{Introduction}

\subsection{Jack polynomials.}

Jack polynomials $\left(J_{\pi}^{(\alpha)}\right)$ are a family of symmetric functions that depend on a parameter $\alpha>0$ and is indexed by an integer partition $\pi$. They were introduced by Henry Jack in his seminal paper [Jac71]. For certain values of $\alpha$, Jack polynomials coincide with various well-known symmetric polynomials. For instance, up to multiplicative constants,

${ }^{*}$ Research supported by Narodowe Centrum Nauki, grant number 2014/15/B/ST1/00064 
Jack polynomials coincide with Schur polynomials for $\alpha=1$; with the zonal polynomials, for $\alpha=2$; with the symplectic zonal polynomials, for $\alpha=1 / 2$; with the elementary symmetric functions, for $\alpha=0$; and in some sense with the monomial symmetric functions, for $\alpha=\infty$. Since it has been shown that several results concerning Schur and zonal polynomials can be generalized in a rather natural way to Jack polynomials [Mac15, Section (VI.10)], Jack polynomials can be viewed as a natural interpolation between several interesting families of symmetric functions.

Connections of Jack polynomials with various fields of mathematics and physics were established: it turned out that they play a crucial role in understanding Ewens random permutations model [DH92], generalized $\beta$-ensembles and some statistical mechanics models [OO97], Selberg-type integrals [Kan93], certain random partition models [Ker00], and some problems of the algebraic geometry [Nak96, Oko03], among many others. Better understanding of Jack polynomials is also very desirable in the context of generalized $\beta$-ensembles and their discrete counterpart model [OO97]. Jack polynomials are a special case of the Macdonald polynomials [Sta89, Mac15].

\subsection{Connection coefficients for Jack symmetric functions.}

Goulden and Jackson [GJ96] defined two families of coefficients $\left(c_{\pi, \sigma}^{\lambda}\right)$ and $\left(h_{\pi, \sigma}^{\lambda}\right)$ depending implicitly on the deformation parameter $\alpha$ and indexed by triples of integer partitions $\pi, \sigma, \lambda \vdash n$ of the same integer $n$. These coefficients are given by expansions of the left-hand sides in terms of the power-sum symmetric functions:

$$
\sum_{\theta \in \mathcal{P}} \frac{1}{\left\langle J_{\theta}, J_{\theta}\right\rangle_{\alpha}} J_{\theta}^{(\alpha)}(\mathbf{x}) J_{\theta}^{(\alpha)}(\mathbf{y}) J_{\theta}^{(\alpha)}(\mathbf{z}) t^{|\theta|}=\sum_{n \geqslant 1} t^{n} \sum_{\lambda, \pi, \sigma \vdash n} \frac{c_{\pi, \sigma}^{\lambda}}{\alpha^{\ell(\lambda)}} z_{\lambda}^{-1} p_{\pi}(\mathbf{x}) p_{\sigma}(\mathbf{y}) p_{\lambda}(\mathbf{z}),
$$

and

$$
\begin{aligned}
\alpha t \frac{\partial}{\partial t} \log \left(\sum_{\theta \in \mathcal{P}} \frac{1}{\left\langle J_{\theta}, J_{\theta}\right\rangle_{\alpha}} J_{\theta}^{(\alpha)}(\mathbf{x}) J_{\theta}^{(\alpha)}(\mathbf{y}) J_{\theta}^{(\alpha)}(\mathbf{z}) t^{|\theta|}\right) & = \\
& \sum_{n \geqslant 1} t^{n} \sum_{\lambda, \pi, \sigma \vdash n} h_{\pi, \sigma}^{\lambda} p_{\pi}(\mathbf{x}) p_{\sigma}(\mathbf{y}) p_{\lambda}(\mathbf{z}),
\end{aligned}
$$

see Section 2.2 for details of the notation.

Dołega and Féray showed that the connection coefficients $\left(c_{\pi, \sigma}^{\lambda}\right)$ are polynomials in the variable $\beta:=\alpha-1$ with rational coefficients and proved the following upper bound on the degrees of these polynomials [DF16, Proposition B.2.]:

$$
\operatorname{deg}_{\beta} c_{\pi, \sigma}^{\lambda} \leqslant d(\pi, \sigma ; \lambda),
$$

where

$$
d(\pi, \sigma ; \lambda):=(|\pi|-\ell(\pi))+(|\sigma|-\ell(\sigma))-(|\lambda|-\ell(\lambda))
$$

One may wonder of the use of the new variable $\beta$, but this shift seems to be the adequate one in order to look at the connection coefficients from the combinatorial point of view. 


\section{$1.3 \quad$ Jack characters}

We expand Jack polynomial in the basis of power-sum symmetric functions:

$$
J_{\lambda}^{(\alpha)}=\sum_{\mu} \theta_{\mu}^{(\alpha)}(\lambda) p_{\mu}
$$

The above sum runs over partitions $\mu$ such that $|\mu|=|\lambda|$. The coefficient $\theta_{\mu}^{(\alpha)}(\lambda)$ is called unnormalized Jack character.

Jack characters $\theta_{\mu}^{(\alpha)}$ provide a kind of dual information about the Jack polynomials. This kind of approach may be traced back to the work of Kerov and Olshanski [KO94]. For a fixed conjugacy class $\mu$ they considered characters of the symmetric group evaluated on $\mu$. This is opposite to the usual way of viewing the characters of the symmetric groups, namely to fix the representation $\lambda$ and to consider the character as a function of the conjugacy class $\mu$. Lassalle [Las08, Las09] adapted Kerov's and Olshanski's idea to the framework of Jack characters.

As Jack symmetric functions $\left(J_{\lambda}^{(\alpha)}\right)_{\lambda}$ form a basis of the symmetric functions, the functions $\left(\theta_{\mu}^{(\alpha)}\right)_{\mu \vdash n}$ form a basis of the algebra of functions on Young diagrams with $n$ boxes [Fér12, Proposition 4.1]. Dołęa and Féray [DF16, Appendix B.2] showed that the coefficients appearing in the expansion of a pointwise product of two unnormalized Jack characters in the unnormalized Jack character basis coincide with the connection coefficients from (1), namely

$$
\theta_{\pi}^{(\alpha)} \cdot \theta_{\sigma}^{(\alpha)}=\sum_{\mu \vdash n} c_{\pi, \sigma}^{\mu} \theta_{\mu}^{(\alpha)}
$$

for all triples of partitions $\pi, \sigma, \mu \vdash n$. This observation encourages us to look more closely into the field of connection coefficients via the context of Jack characters.

Better understanding of the combinatorics of Jack characters leads to a better understanding of Jack polynomials themselves. In particular, we found the form of the leading coefficient of $c_{\pi, \sigma}^{\mu}$ be investigating properties of Jack characters.

\subsection{Matchings.}

We present the well established terminology of matchings given in [GJ96]. For a given integer $n$ we consider the following set

$$
\mathcal{N}_{n}=\{1, \hat{1}, \ldots, n, \hat{n}\}
$$

We denote by $\mathcal{F}_{n}$ the set of all matchings (partitions into two-element sets) on $\mathcal{N}_{n}$. For matchings $\delta_{1}, \delta_{2}, \ldots \in \mathcal{F}_{n}$ we denote by $G\left(\delta_{1}, \delta_{2}, \ldots\right)$ the multi-graph with the vertex set $\mathcal{N}_{n}$ whose edges are formed by the pairs in $\delta_{1}, \delta_{2}, \ldots$. For given matchings $\delta_{1}, \delta_{2}$ the corresponding graph $G\left(\delta_{1}, \delta_{2}\right)$ consists of disjoint even cycles, since each vertex has degree 2 and around each cycle the edges alternate between $\delta_{1}$ and $\delta_{2}$. Denote by $\Lambda\left(\delta_{1}, \delta_{2}\right)$ the partition of $n$ which specifies halves the lengths of the cycles in $G\left(\delta_{1}, \delta_{2}\right)$. More generally, 


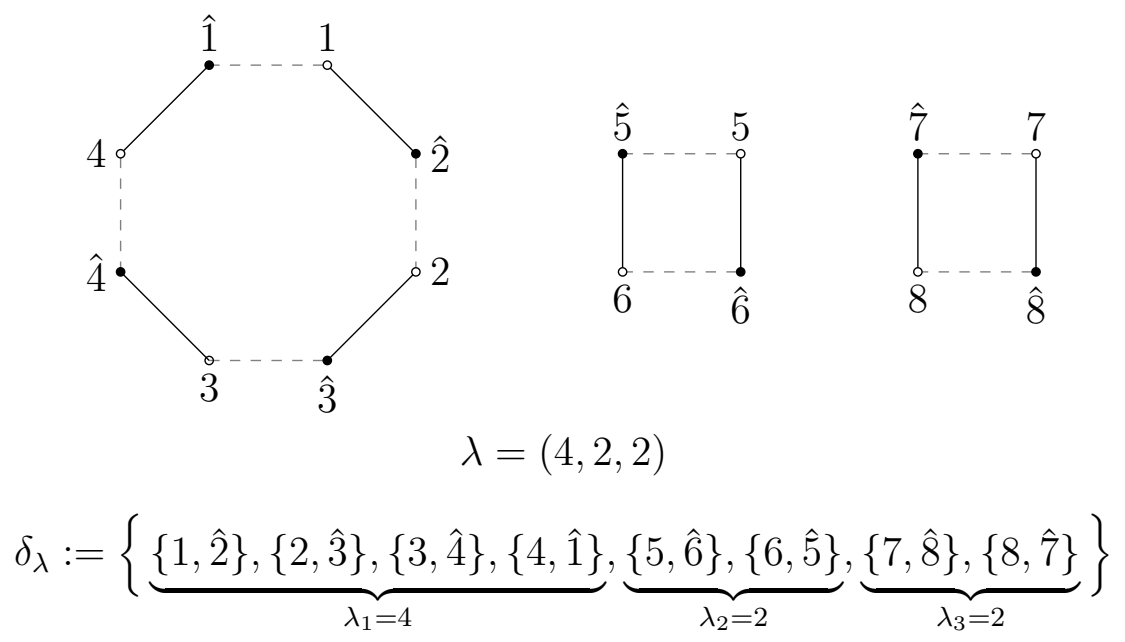

Figure 1 - An example of bipartite matchings $\epsilon$ (dotted line) and $\delta_{\lambda}$ (continuous line) associated with $\lambda=(4,2,2)$. Notice that $\Lambda\left(\epsilon, \delta_{\lambda}\right)=\lambda$.

denote by $\Lambda\left(\delta_{1}, \ldots, \delta_{s}\right)$ the partition of $n$ which specifies halves of the number of vertices in each connected component of $G\left(\delta_{1}, \delta_{2}, \ldots\right)$ (it is an easy observation that such numbers form a partition of $n$ ).

We call the sets $\{1, \ldots, n\}$ and $\{\hat{1}, \ldots, \hat{n}\}$ classes of $\mathcal{N}_{n}$. A pair in a matching is called a between-class pair if it contains elements of different classes. A matching $\delta$ in which every pair is a between-class pair is called a bipartite matching (in this case $G(\delta)$ is a bipartite graph on the vertex-sets given by the two classes of $\mathcal{N}_{n}$ ).

We introduce the following specific bipartite matchings in the set $\mathcal{F}_{n}$. Firstly, let

$$
\epsilon:=\{\{1, \hat{1}\}, \ldots,\{n, \hat{n}\}\}
$$

secondly, for a given partition $\lambda \vdash n$, let

$$
\begin{aligned}
\delta_{\lambda}:=\left\{\{1, \hat{2}\},\{2, \hat{3}\}, \ldots,\left\{\lambda_{1}-1, \hat{\lambda_{1}}\right\},\left\{\lambda_{1}, \hat{1}\right\},\right. \\
\left.\left\{\lambda_{1}+1, \lambda_{1} \hat{+} 2\right\}, \ldots,\left\{\lambda_{1}+\lambda_{1}-1, \lambda_{1} \hat{+} \lambda_{1}\right\},\left\{\lambda_{1}+\lambda_{1}, \lambda_{1} \hat{+} 1\right\}, \ldots\right\},
\end{aligned}
$$

see Figure 1. Observe that both matchings $\epsilon$ and $\delta_{\lambda}$ are bipartite and $\Lambda\left(\epsilon, \delta_{\lambda}\right)=\lambda$.

\subsection{Matchings-Jack Conjecture.}

Definition 1.1. For given three partitions $\pi, \sigma, \lambda \vdash n$, we denote by $\mathcal{G}_{\pi, \sigma}^{\lambda}$ the set of all matchings $\delta \in \mathcal{F}_{n}$, for which $\Lambda(\delta, \epsilon)=\pi$ and $\Lambda\left(\delta, \delta_{\lambda}\right)=\sigma$.

Goulden and Jackson observed [GJ96, Proposition 4.1] that the specializations of $c_{\pi, \sigma}^{\lambda}(\beta)$ for $\beta \in\{0,1\}$ may be expressed in terms of matchings, namely

$$
\begin{aligned}
& c_{\pi, \sigma}^{\lambda}(0)=\mid\left\{\delta \in \mathcal{G}_{\pi, \sigma}^{\lambda}: \delta \text { is bipartite }\right\} \mid, \\
& c_{\pi, \sigma}^{\lambda}(1)=\left|\left\{\delta \in \mathcal{G}_{\pi, \sigma}^{\lambda}\right\}\right| .
\end{aligned}
$$


In fact, those specialisations coincide with the connection coefficients of two commutative subalgebras of the group algebra of the symmetric group: the class algebra and the double coset algebra ( $\beta=0$ and $\beta=1$ respectively) [HSS92].

Based on this observation Goulden and Jackson conjectured that the family $\left(c_{\pi, \sigma}^{\lambda}\right)$ of polynomials may have a combinatorial interpretation. The conjecture is known as the Matchings-Jack Conjecture.

Conjecture 1.2 (Matchings-Jack Conjecture). For any partitions $\pi, \sigma, \lambda \vdash n$ the quantity $c_{\pi, \sigma}^{\lambda}$ can be expressed as

$$
c_{\pi, \sigma}^{\lambda}(\beta)=\sum_{\delta \in \mathcal{G}_{\pi, \sigma}^{\lambda}} \beta^{\mathrm{wt}_{\lambda}(\delta)}
$$

where $\mathrm{wt}_{\lambda}: \mathcal{G}_{\pi, \sigma}^{\lambda} \longrightarrow \mathbb{N}$ is some hypothetical combinatorial statistic, which vanishes if and only if $\delta$ is bipartite.

Clearly, it seems that the statistic $\mathrm{wt}_{\lambda}$ should be a marker of non-bipartiteness for matchings. Matchings-Jack Conjecture remains still open in the general case, however some special cases have been settled. Goulden and Jackson constructed some statistics $\mathrm{wt}_{\lambda}$ for $\lambda=\left[1^{n}\right]$ and $\lambda=\left[2,1^{n-1}\right]$ and proved the conjecture in those cases [GJ96]. Later on, the Matchings-Jack Conjecture has been proved by Kanunnikov and Vassiliveva [KV16] in the case $\pi=\sigma=(n)$ of the partitions with exactly one part. Recently, in a joint paper with Promyslov [KVP17], they proved a variation of this conjecture, which involves additional labellings on matchings, in the special case when one of the three partitions is equal to $(n)$. They made use of the measure of non-orientability $\theta$ defined by La Croix in his $\mathrm{PhD}$ thesis [La 09]. The measure of non-orientability $\theta$ is a statistic defined on a class of rooted maps. In some special cases it may be translated into the field of matchings, however generally significant difficulties appear. We also shall use the same statistic.

\subsection{The main result.}

In the current paper we give a necessary and sufficient condition for the polynomial $c_{\pi, \sigma}^{\lambda}$ to achieve the maximal degree given by (3). Moreover, we show that the leading coefficient of $c_{\pi, \sigma}^{\lambda}$ of this maximal degree is a non-negative integer and we present it in the context of Matchings-Jack Conjecture.

Definition 1.3. Consider two integer partitions $\lambda$ and $\mu$ of the same integer $n$, let $k=\ell(\lambda)$ and $m=\ell(\mu)$ be the lengths of the partitions. We say that $\lambda$ is a sub-partition of $\mu$ (denoted $\lambda \preceq \mu$ ) if there exists a set-partition $\nu$ of $[k]$, such that

$$
\mu_{i}=\sum_{j \in \nu_{i}} \lambda_{j}
$$

for any $i \in[m]$, see Figure 2. We denote $\lambda \prec \mu$ if $\lambda \preceq \mu$ and $\lambda \neq \mu$.

In addition, consider the third partition $\pi$ of the same integer $n$ of the length $l=\ell(\pi)$. We say that $\lambda$ and $\pi$ are joint sub-partitions of $\mu$ if there exist set-partitions $\nu$ of $[k]$ and $\tau$ of $[l]$ such that

$$
\mu_{i}=\sum_{j \in \nu_{i}} \lambda_{j}=\sum_{j \in \tau_{i}} \pi_{j}
$$



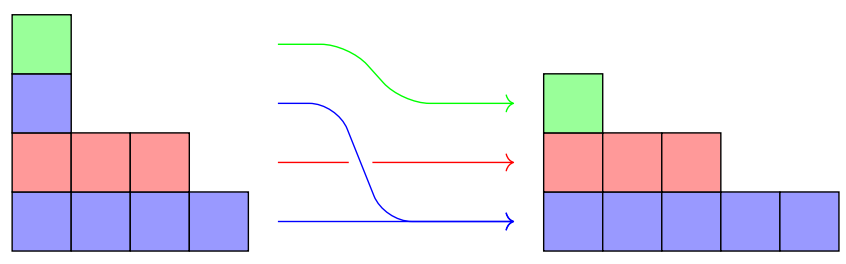

Figure $2-$ A pair of partitions $\lambda=(4,3,1,1)$ and $\mu=(5,3,1)$ presented as Young diagrams. Partition $\lambda$ is sub-partition of $\mu$; indeed, each part of $\mu$ is given as a sum of different parts of $\lambda$.

and moreover

$$
\left|\nu_{i}\right|+\left|\tau_{i}\right| \leqslant \mu_{i}+1
$$

for all $i \in[m]$.

Definition 1.4. For given partitions $\pi, \sigma, \lambda, \mu \vdash n$, we denote by $\mathcal{G}_{\pi, \sigma}^{\lambda ; \mu}$ the set of all matchings $\delta \in \mathcal{G}_{\pi, \sigma}^{\lambda}$ which are $\mu$-connected, i.e. $\Lambda\left(\delta, \epsilon, \delta_{\lambda}\right)=\mu$.

The class $\mathcal{G}_{\pi, \sigma}^{\lambda}$ splits naturally into the classes $\mathcal{G}_{\pi, \sigma}^{\lambda ; \mu}$, namely

$$
\mathcal{G}_{\pi, \sigma}^{\lambda}=\bigsqcup_{\mu: \lambda \preceq \mu} \mathcal{G}_{\pi, \sigma}^{\lambda ; \mu}
$$

Contrary to previous works on the Matchings-Jack Conjecture we do not attempt to define the statistic $\mathrm{wt}_{\lambda}$ on $\mathcal{G}_{\pi, \sigma}^{\lambda}$ for a particular class of partitions $\lambda, \pi$ or $\sigma$. We define the statistic "stat" on the class $\mathcal{G}_{\pi, \sigma}^{\lambda ; \lambda}$.

Theorem 1.5 (The main result). For any triple of partitions $\pi, \sigma, \lambda \vdash n$ the corresponding polynomial $c_{\pi, \sigma}^{\lambda}(\beta)$ achieves the upper bound on the degree given in (3) if and only if $\pi$ and $\sigma$ are joint sub-partitions of $\mu$. For such partitions, the leading coefficient of $c_{\pi, \sigma}^{\lambda}(\beta)$ may be expressed in two different manners:

$$
\begin{aligned}
& {\left[\beta^{d(\pi, \sigma ; \lambda)}\right] c_{\pi, \sigma}^{\lambda}=\mid \delta \in \mathcal{G}_{\pi, \sigma}^{\lambda ; \lambda}: \delta \text { is unhandled } \mid=} \\
& \qquad \sum_{\nu: \nu \preceq \lambda} \frac{z_{\lambda}}{z_{\nu}} \mid \delta \in \mathcal{G}_{\pi, \sigma}^{\nu ; \lambda}: \delta \text { is bipartite } \mid,
\end{aligned}
$$

for notion of unhandled matchings see Definition 5.11, scaling factors $z_{\lambda}, z_{\nu}$ are defined in Section 2. Moreover, there exists a statistic stat $: \mathcal{G}_{\pi, \sigma}^{\lambda ; \lambda} \longrightarrow \mathbb{N}_{0}$, which satisfies

$$
\left[\beta^{d(\pi, \sigma ; \lambda)}\right] c_{\pi, \sigma}^{\lambda}=\left[\beta^{d(\pi, \sigma ; \lambda)}\right] \sum_{\delta \in \mathcal{G}_{\pi, \sigma}^{\lambda ; \lambda}} \beta^{\operatorname{stat}(\delta)}
$$

and for $\delta \in \mathcal{G}_{\pi, \sigma}^{\lambda ; \lambda}$ the statistic $\operatorname{stat}(\delta)$ vanishes if and only if $\delta$ is bipartite. 


\subsection{Organisation of the paper.}

In Section 3, we introduce the terminology of maps and we investigate relations between maps and matchings. We made use of this terminology in Section 4, where we define normalized Jack characters and their structure constants. Structure constants play a central role in our paper. In particular, in Section 4 we provide an explicit formula for the top-degree part of structure constants of Jack characters, see Theorem 4.3. Only after, in Section 6 we show the relation between the structure constants for Jack characters and the connection coefficients for Jack symmetric functions. We translate obtained results for the top-degree part of structure constants into the language of connection coefficients there. In Section 5 we present a measure of non-orientability in the context of the $b$ Conjecture. We present a problem of transferring it into the satisfactory statistic which measures non-bipartitness of a matching. We discuss the recent result of Dołęga [Doł17] about the top-degree part in the $b$-Conjecture. Our result and the result of Dołega are in some sense equivalent, see Section A, however, the methods used in both papers are different.

\section{Preliminaries}

\subsection{Partitions.}

A partition $\lambda$ of an integer $n$ (denoted by $\lambda \vdash n$ ) is a non-increasing list $\left(\lambda_{1}, \ldots, \lambda_{l}\right)$ of positive integers of sum equal to $n$. Number $n$ is called the size of $\lambda$ and is denoted by $|\lambda|$, the number $l$ is the length of the partition, denoted by $\ell(\lambda)$. Finally,

$$
m_{i}(\lambda):=\left|\left\{k: \lambda_{k}=i\right\}\right|
$$

is the multiplicity of $i \geqslant 1$ in the partition $\lambda$.

There are many orders on the set of partitions. Beside the one shown in Definition 1.3 we introduce the dominance order. We say that $\lambda \leqslant \mu$ if and only if

$$
\sum_{i \leqslant j} \lambda_{i} \leqslant \sum_{i \leqslant j} \mu_{i}
$$

holds for any positive integer $j$.

For given two partitions $\lambda$ and $\mu$ we construct their concatenation (denoted $\lambda \cup \mu$ ) by merging all parts from $\lambda$ and $\mu$ and ordering them in a decreasing fashion.

\subsection{Jack polynomials.}

Let us consider the vector space $\Lambda_{\mathbb{Q}(\alpha)}$ of the symmetric functions [Mac15] over the field of rational functions $\mathbb{Q}(\alpha)$ and its basis $\left(p_{\lambda}\right)_{\lambda}$ of power-sum symmetric functions, i.e. the symmetric functions given by

$$
p_{\lambda}(\mathbf{x})=\prod_{i} p_{\lambda_{i}}(\mathbf{x}), \quad p_{k}(\mathbf{x})=x_{1}^{k}+x_{2}^{k}+\cdots .
$$


The following scalar product on $\Lambda_{\mathbb{Q}(\alpha)}$ is defined on the power-sum basis by the formula

$$
\left\langle p_{\lambda}, p_{\mu}\right\rangle_{\alpha}:=\alpha^{\ell(\lambda)} z_{\lambda} \delta_{\lambda, \mu}
$$

where

$$
z_{\lambda}=\prod_{i} i^{m_{i}(\lambda)} m_{i}(\lambda) !
$$

and further extended by bilinearity. This is a classical deformation of the Hall inner product, which corresponds to $\alpha=1$ [Jac71].

The family of Jack polynomials $\left(J_{\pi}^{(\alpha)}\right)$ is the unique family of symmetric functions which satisfies the following three criteria:

1. $J_{\lambda}^{(\alpha)}=\sum_{\mu \leqslant \lambda} a_{\mu}^{\lambda} m_{\mu}$, where $a_{\mu}^{\lambda} \in \mathbb{Q}[\alpha]$,

2. $\left[m_{1|\lambda|}\right] J_{\lambda}^{(\alpha)}:=a_{1|\lambda|}^{\lambda}=|\lambda| !$,

3. $\left\langle J_{\lambda}^{(\alpha)}, J_{\mu}^{(\alpha)}\right\rangle_{\alpha}=0$ for $\lambda \neq \mu$,

where $m_{\lambda}$ denotes the monomial symmetric function associated with $\lambda$.

\section{Matchings and maps}

\subsection{Maps.}

In the literature a map [LZ04] is classically defined as a connected graph $G$ (possibly, with multiple edges) drawn on a surface $\Sigma$, i.e. a compact connected 2-dimensional manifold without boundary. We assume that the collection of faces $($ i.e. $\Sigma \backslash \mathcal{E}$ ) is homeomorphic to the collection of open discs. A choice of an edge-side and one of its endpoints is called a root of the map, see Figure 3. A map together with a choice of a root is called a rooted map.

A vertex two-coloured map is called bipartite if each edge connects vertices of different colors; for simplicity we set that there are white and black vertices, we denote by $\mathcal{W}(\mathcal{B})$ the set of white (black) vertices. By convention, from a rooted bipartite map we require that the root vertex is black. Figure 3 presents an example of a rooted bipartite map $M$. With a given bipartite map $M$ with $n$ edges we associate two integer partitions of $n$ :

$$
\Lambda_{\mathcal{W}}(M) \text { and } \Lambda_{\mathcal{B}}(M)
$$

given by the degrees of white/black vertices. For such a map we assign also the third partition

$$
\Lambda_{\mathcal{F}}(M)
$$

of $n$, which describes the face structure of $M$; it is specified by reading halves of the numbers of edge-sides fencing each face (since the map $M$ is bipartite, for each face there is an even number of edges adjacent to the face). The partition $\Lambda_{\mathcal{F}}(M)$ is called the face-type of the map $M$, see Figure 3 . 
Definition 3.1. For three given partitions $\pi, \sigma, \lambda \vdash n$ we denote by $M_{\pi, \sigma}^{\bullet}$ the set of all bipartite, rooted maps $M$ with $n$ edges, for which $\Lambda_{\mathcal{W}}(M)=\pi$ and $\Lambda_{\mathcal{B}}(M)=\sigma$. Moreover we denote by $M_{\pi, \sigma}^{\lambda}$ the set of all such maps $M$ which additionally have the face-type $\lambda$, i.e. $\Lambda_{\mathcal{F}}(M)=\lambda$.

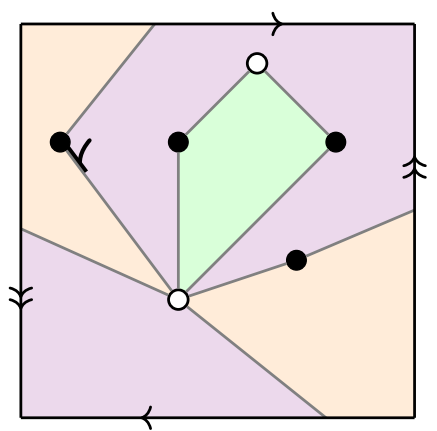

$$
\begin{aligned}
& \Lambda_{\mathcal{F}}(M)=(4,2,2) \\
& \Lambda_{\mathcal{W}}(M)=(6,2) \\
& \Lambda_{\mathcal{B}}(M)=(2,2,2,2)
\end{aligned}
$$

Figure 3 - Example of a rooted bipartite map $M$ on the projective plane. The left side of the square should be glued to the right side, as well as bottom to top, as indicated by the arrows. We present also the face, white and black vertex distributions.

Due to the nature of our result we extend this definition slightly, namely we waive the assumption of connectedness in the definition of a map. There are two natural ways to generalize the notion of connected maps to non-connected ones: either we consider lists of connected maps or we consider collections of them.

\subsection{Lists and collections of maps.}

Definition 3.2. Let $\mu=\left(\mu_{1}, \ldots, \mu_{k}\right)$ be a partition of an integer $n$. A list of maps $\left(M_{1}, \ldots, M_{k}\right)$ is called a $\mu$-list of maps if the map $M_{i}$ has $\mu_{i}$ edges for each $i \in[k]$. We say that such a list is rooted, respectively bipartite if each map $M_{i}$ is so. For a bipartite $\mu$-list of maps we associate three partitions describing the black vertex, the white vertex and the face structures

$$
\Lambda_{\mathcal{W}}(M):=\bigcup_{i=1}^{k} \Lambda_{\mathcal{W}}\left(M_{i}\right), \quad \Lambda_{\mathcal{B}}(M):=\bigcup_{i=1}^{k} \Lambda_{\mathcal{B}}\left(M_{i}\right), \quad \Lambda_{\mathcal{F}}(M):=\bigcup_{i=1}^{k} \Lambda_{\mathcal{F}}\left(M_{i}\right),
$$

where $U$ denotes the concatenation of partitions.

Definition 3.3 (Extension of Definition 3.1). For given partitions $\pi, \sigma, \mu \vdash n$, we denote by $M_{\pi, \sigma}^{\bullet ; \mu}$ the set of all bipartite rooted $\mu$-lists of maps $M$ which satisfy

$$
\Lambda_{\mathcal{W}}(M)=\pi \quad \text { and } \quad \Lambda_{\mathcal{B}}(M)=\sigma,
$$

see Figure 4. Moreover, for a given partition $\lambda \vdash n$ we denote by $M_{\pi, \sigma}^{\lambda ; \mu}$ the set of all $\mu$-lists of maps $M \in M_{\pi, \sigma}^{\bullet ; \mu}$ which have face-type $\lambda$, i.e. $\Lambda_{\mathcal{F}}(M)=\lambda$.

Definition 3.4. Let $\mu=\left(\mu_{1}, \ldots, \mu_{k}\right)$ be a partition of an integer $n$. A multiset of maps $\left\{M_{1}, \ldots, M_{k}\right\}$ is called a $\mu$-collection of maps if the map $M_{i}$ has $\mu_{i}$ edges for each $i \in[k]$. 


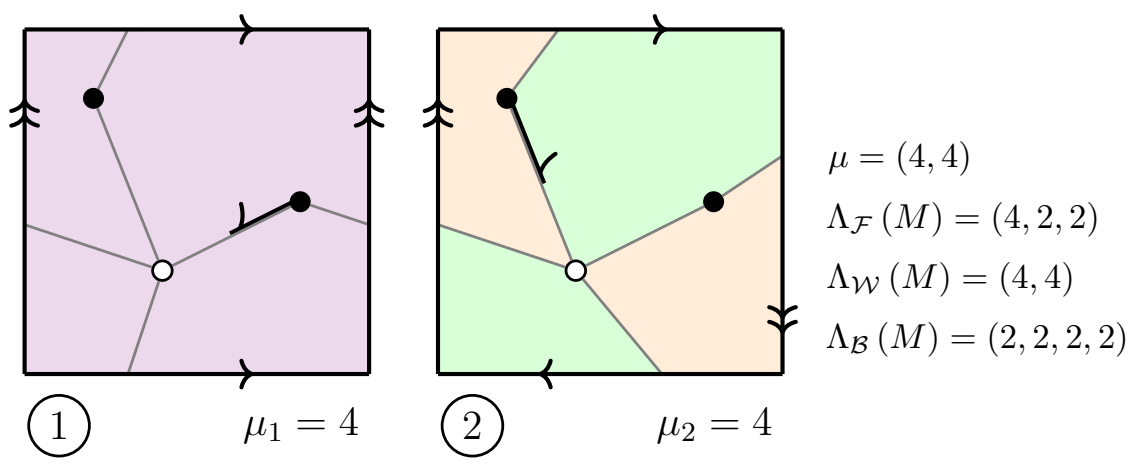

Figure 4 - Example of a rooted, bipartite $\mu$-list of maps for the partition $\mu=(4,4)$. The first map is drawn on the torus, the second one on the projective plane. We present also the face, white vertices and black vertices distributions. By erasing the roots and the numbering of the connected components we obtain a bipartite $\mu$-collection of maps.

We say that such a collection is rooted or bipartite if each map $M_{i}$ is so. For such a collection of maps we associate three partitions describing black, white and face structures as in Definition 3.2.

Roughly speaking, a $\mu$-collection of maps could be created from a $\mu$-list of maps by erasing the numbering of the connected components, i.e. the order on the connected components (see Figure 4).

\subsection{Matchings and maps.}

Matchings and maps are closely related notions. Roughly speaking, a bipartite matching can be treated as a (possibly non-connected) bipartite map with rooted and numbered faces. We shall discuss relations between matchings and rooted list of maps with the same face, black vertices and white vertices distributions.

Definition 3.5. Consider two partitions $\lambda, \mu \vdash n$. We say that a bipartite $\mu$-collection $M$ of maps with the face distribution given by $\lambda$ has rooted and numbered faces if all faces of $M$ are rooted (i.e. on each face there is one marked edge-side) and each face is labelled by a distinct number $i \in[\ell(\lambda)]$ in such way that it is surrounded by $2 \lambda_{i}$ edges, see Figure 5. The set of such collections of maps with the face, black vertices and white vertices distributions given by the partitions $\lambda, \pi, \sigma \vdash n$ is denoted by $M\left(\mathcal{G}_{\pi, \sigma}^{\lambda ; \mu}\right)$.

Remark 3.6. Observe that rooting a face is nothing else but choosing one of the face corners adjacent to some black vertex and orienting the face. Through a map (or a list/a collection of maps) with rooted faces we can understand a map with oriented faces and chosen black corners for each of the faces, see Figure 5. Similarly, rooting a map is choosing one corner of a black vertex and orienting the face adjacent to this corner.

We consider four partitions: $\pi, \sigma, \lambda, \mu \vdash n$. To a given matching $\delta \in \mathcal{G}_{\pi, \sigma}^{\lambda ; \mu}$ we associate a bipartite $\mu$-collection $M_{\delta} \in M\left(\mathcal{G}_{\pi, \sigma}^{\lambda ; \mu}\right)$ given by the following procedure. 

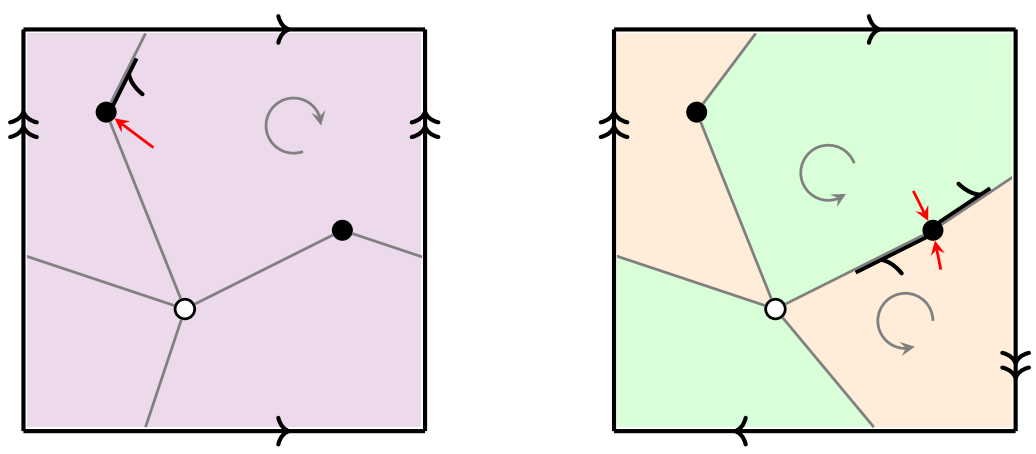

Figure 5 - Example of a bipartite $(4,4)$-collection of maps with rooted faces. By rooting faces we understand choosing one edge-side of each face (drawn as a black half-arrow going from a black vertex) or, equivalently, orienting each face (the rounded arrows) and choosing one black vertex for each face (the red arrows).

1. The matchings $\epsilon$ and $\delta_{\lambda}$ determine the polygons with the vertices labelled by $\mathcal{N}_{n}$, see Figure 1. We take theirs duals, i.e. the polygons with the edges labelled by $\mathcal{N}_{n}$, see Figure 6 . The consecutive polygons have $2 \lambda_{1}, 2 \lambda_{2}, \ldots$ edges. Observe that the parts of $\epsilon$ (respectively $\delta_{\lambda}$ ) can be identified with the black (respectively white) vertices as it is shown on Figure 6;

2. The matching $\delta$ determines the unique way of gluing the edges of the polygons in such a way that black (white) vertices are glued with black (white) ones. Figure 7 presents such a gluing for the matching

$$
\delta=\{\{\hat{1}, 6\},\{1, \hat{6}\},\{\hat{2}, \hat{7}\},\{2,7\},\{\hat{3}, \hat{8}\},\{3,8\},\{\hat{4}, 5\},\{4, \hat{5}\}\} .
$$

Observe that the distribution of black (respectively white) vertices is given by $\Lambda(\delta, \epsilon)$ (respectively by $\Lambda\left(\delta, \delta_{\lambda}\right)$ ). Moreover, $\mu=\Lambda\left(\delta, \epsilon, \delta_{\lambda}\right)$.

3. Each face is canonically numbered by an integer $s$ related to the polygon $\lambda_{s}$, i.e. the edge-sides of this face are labelled by the elements

$$
\sum_{i=1}^{s-1} \lambda_{i}+1, \ldots, \sum_{i=1}^{s-1} \lambda_{i}+\lambda_{s}, \widehat{s-\widehat{s} \lambda_{i=1}}+1, \ldots \widehat{\sum_{i=1}^{s-\widehat{1}} \lambda_{i}+\lambda_{s}} .
$$

Such a face is canonically rooted by selecting the edge-side labelled by the number $\sum_{i=1}^{s-1} \lambda_{i}+1$, see Figure 7 .

4. We remove the labelling by the elements from $\mathcal{N}_{n}$.

Corollary 3.7. The procedure described above gives a bijection $\delta \mapsto M_{\delta}$ between the set of matchings $\mathcal{G}_{\pi, \sigma}^{\lambda ; \mu}$ and the set of collections of maps $M\left(\mathcal{G}_{\pi, \sigma}^{\lambda ; \mu}\right)$.

We compare the terminologies of matchings and maps in the table below. 

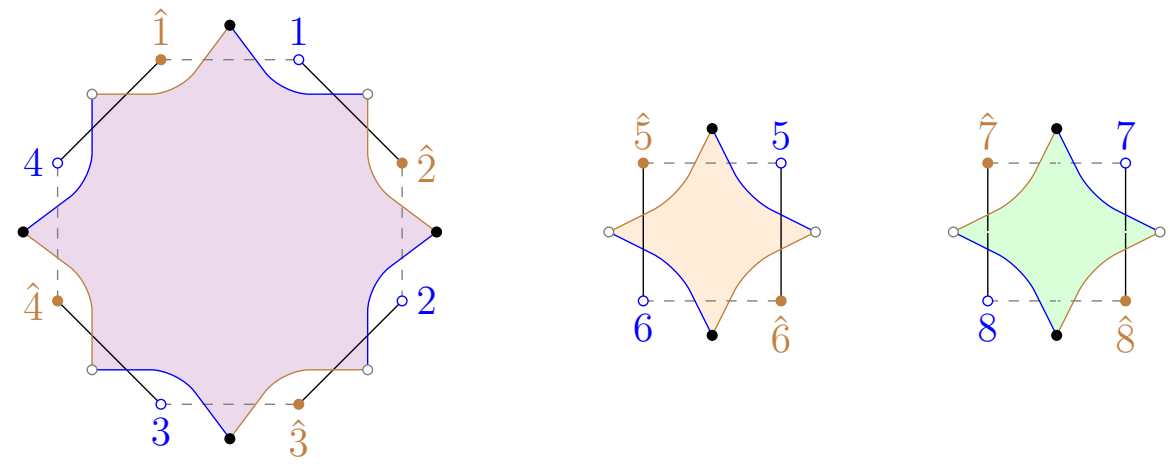

Figure 6 - Duals of the polygons created by the matchings $\epsilon$ and $\delta_{\lambda}$ presented on Figure 1 . Black (respectively white) vertices of such polygons are labelled by the elements of $\epsilon$ (respectively $\delta_{\lambda}$ ), the edges by the elements from $\mathcal{N}_{8}$.

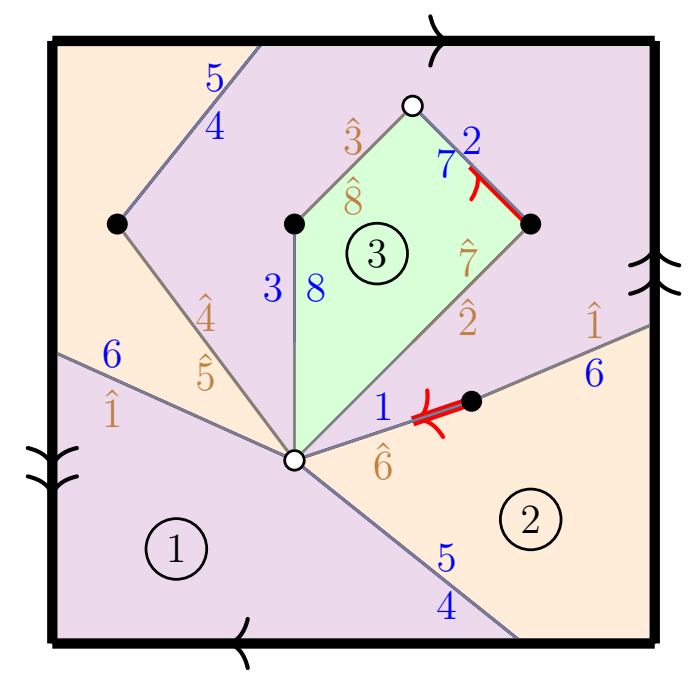

$$
\delta=\{\{\hat{1}, 6\},\{1, \hat{6}\},\{\hat{2}, \hat{7}\},\{2,7\},\{\hat{3}, \hat{8}\},\{3,8\},\{\hat{4}, \hat{5}\},\{4,5\}\}
$$

Figure 7 - Matching $\delta$ on the set $\mathcal{N}_{8}$ describes the way of gluing the sides of the polygons from Figure 6. Labels from $\mathcal{N}_{8}$ determine the way of numbering and rooting faces of such a map (in general it could be a collection of maps), the roots (presented as half-arrows) correspond to the labels $1,5,7$. Numbers of faces are presented in black circles. 


\begin{tabular}{|l|c|c|}
\hline & Matching $\delta$ & $\mu$-list of maps $M$ \\
\hline face-type & $\lambda$ & $\Lambda_{\mathcal{F}}(M)$ \\
distribution of black vertices & $\Lambda(\delta, \epsilon)$ & $\Lambda_{\mathcal{B}}(M)$ \\
distribution of white vertices & $\Lambda\left(\delta, \delta_{\lambda}\right)$ & $\Lambda_{\mathcal{W}}(M)$ \\
connected components & $\Lambda\left(\delta_{\lambda}, \epsilon, \delta\right)$ & $\mu$ \\
$\mu$-collections of maps with given faces, & $\mathcal{G}_{\pi ; \mu}^{\lambda ; \mu}$ & $M\left(\mathcal{G}_{\pi, \sigma}^{\lambda ; \mu}\right)$ \\
black and white vertices distribution & & \\
and with rooted and numbered faces & & \\
\hline
\end{tabular}

\subsection{Matchings and lists of rooted maps.}

We showed that matchings are equivalent to collections of maps with rooted and numbered faces. However, collections of maps with rooted and numbered connected components (i.e. lists of rooted maps) are much more natural objects. We give a relation between those two ways of numbering and rooting collections of maps. More precisely, we present a relation between the set $M\left(\mathcal{G}_{\pi, \sigma}^{\lambda ; \mu}\right)$ and the set $M_{\pi, \sigma}^{\lambda ; \mu}$.

What is common for those two classes is the fact that by rooting and numbering faces or connected components, the group of automorphisms becomes trivial.

Definition 3.8. For a given $\mu$-collection of maps with rooted and numbered faces $M \in$ $M\left(\mathcal{G}_{\pi, \sigma}^{\lambda ; \mu}\right)$ we define the set $\mathcal{R}(M)$ of all numberings of the connected components and rooting each of them in such a way that with respect to them $M$ becomes a $\mu$-list of maps from $M_{\pi, \sigma}^{\lambda ; \mu}$. We call $\mathcal{R}(M)$ the set of components-labellings of $M$. For a given $r \in \mathcal{R}(M)$ we denote $(M, r) \in M_{\pi, \sigma}^{\lambda ; \mu}$.

Similarly, for a given $\mu$-list of maps $M \in M_{\pi, \sigma}^{\lambda ; \mu}$ we define the set $\mathcal{L}(M)$ of all numberings of the faces and rooting each of them in such a way that $M$ becomes an element from $M\left(\mathcal{G}_{\pi, \sigma}^{\lambda ; \mu}\right)$. We call $\mathcal{L}(M)$ the set of faces-labellings of $M$. For a given $l \in \mathcal{L}(M)$ we denote $(M, l) \in M\left(\mathcal{G}_{\pi, \sigma}^{\lambda ; \mu}\right)$.

Observation 3.9. Let us fix partitions $\pi, \sigma, \mu, \lambda \vdash n$. For each $M_{1} \in M\left(\mathcal{G}_{\pi, \sigma}^{\lambda ; \mu}\right)$ and $M_{2} \in$ $M_{\pi, \sigma}^{\lambda ; \mu}$ we have

$$
\left|\mathcal{R}\left(M_{1}\right)\right|=2^{\ell(\mu)} z_{\mu} \quad \text { and } \quad\left|\mathcal{L}\left(M_{1}\right)\right|=2^{\ell(\lambda)} z_{\lambda} .
$$

Proof. Let us take $M \in M\left(\mathcal{G}_{\pi, \sigma}^{\lambda ; \mu}\right)$. There are $\prod_{i} m_{i}(\mu)$ ! ways of numbering the connected components and $\prod_{i}(2 i)^{m_{i}(\mu)}$ ways of rooting each of them. We may carry out a similar deduction for $M \in M_{\pi, \sigma}^{\lambda ; \mu}$.

Observation 3.10. For given partitions $\pi, \sigma, \mu, \lambda \vdash n$ we have

$$
\left|\mathcal{G}_{\pi, \sigma}^{\lambda ; \mu}\right|=\left|M\left(\mathcal{G}_{\pi, \sigma}^{\lambda ; \mu}\right)\right|=\frac{z_{\lambda} 2^{\ell(\lambda)}}{z_{\mu} 2^{\ell(\mu)}}\left|M_{\pi, \sigma}^{\lambda ; \mu}\right| .
$$



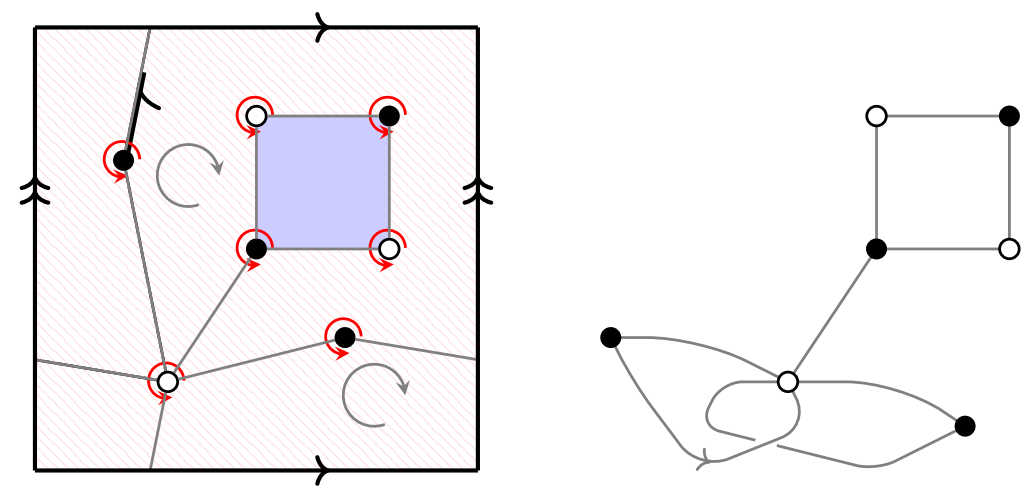

Figure 8 - Example of a rooted orientable map $M$ drawn as a graph on a torus (on the left). There is the canonical orientation (grey arrows) given by the root. We are going to present oriented maps in such a way that their orientation is consistent with the clockwise orientation of the page (grey arrows) or, equivalently, the counter-clockwise orientation around each vertex (red arrows). The distinction between chosen orientations of the page and the vertices may seem awkward. However, it is more convenient for the purpose of Section 5.2. With this convention we can present the root of a map (similarly roots of lists of maps) by an arrow going out from a black vertex. Since $M$ is oriented, it can be recovered from a graphical representation on the plane as a graph with a fixed cyclic order of outgoing edges around each vertex together with a choice of the root (on the right).

Proof. The first equation follows from Corollary 3.7. We investigate the second one. Each collection of maps from $M\left(\mathcal{G}_{\pi, \sigma}^{\lambda ; \mu}\right)$ has rooted and numbered faces, each collection of maps from $M_{\pi, \sigma}^{\lambda ; \mu}$ has rooted and numbered components. From each of them we can get a collection of maps which have rooted and numbered both: faces and components. The number of ways of doing it is given in Observation 3.9. We use the double counting method and conclude the second equation.

\subsection{Orientable maps and bipartite matchings.}

By an orientable map we understand a map which is drawn on an orientable surface. An orientation of a map is given by orienting each face in such a way, that the two edge-sides forming the same edge are oriented in the opposite way. We say that such an orientation of faces is coherent. Orienting any face is equivalent to orienting a map. Observe that a rooted map possesses the canonical orientation given by the root, see Remark 3.6. By a rooted oriented map we understand an orientable map together with the orientation given by the root, see Figure 8.

Definition 3.11. We use the following notation:

$$
\begin{aligned}
\widetilde{M}_{\pi, \sigma}^{\lambda ; \mu}: & =\left\{M \in M_{\pi, \sigma}^{\lambda ; \mu}: M \text { is orientable }\right\}, \\
\widetilde{M}_{\pi, \sigma}^{\bullet ; \mu}: & =\left\{M \in M_{\pi, \sigma}^{\bullet ; \mu}: M \text { is orientable }\right\}, \\
\widetilde{\mathcal{G}}_{\pi, \sigma}^{\lambda ; \mu} & :=\left\{\delta \in \mathcal{G}_{\pi, \sigma}^{\lambda ; \mu}: \delta \text { is bipartite }\right\} .
\end{aligned}
$$


The notion of bipartiteness of a matching is closely related to the notion of orientability. Observation 3.12. For given partitions $\pi, \sigma, \mu, \lambda \vdash n$, we have

$$
\left|\widetilde{\mathcal{G}}_{\pi, \sigma}^{\lambda ; \mu}\right|=\frac{z_{\lambda}}{z_{\mu}}\left|\widetilde{M}_{\pi, \sigma}^{\lambda ; \mu}\right|
$$

Proof. We identify a matching $\delta \in \mathcal{G}_{\pi, \sigma}^{\lambda ; \mu}$ with a collection of maps $M_{\delta} \in M\left(\mathcal{G}_{\pi, \sigma}^{\lambda ; \mu}\right)$ with rooted and numbered faces by the procedure described in Section 3.3. Observe that a bipartite matching corresponds to a collection of oriented maps. Indeed, the orientations of faces given by the edge-sides: $1, \lambda_{1}+1, \ldots$ are coherent. Observation 3.10 gives a relation between collections of maps with rooted and numbered faces and collections of maps with rooted and numbered components (lists of maps). An analysis similar to the one given in Observation 3.10 convinces us that the quantity $2^{\ell(\mu)} \prod_{i} i^{m_{i}(\lambda)}$ specifies the number of manners of rooting the faces in a coherent way and $\prod_{i} m_{i}(\lambda)$ ! specifies the number of manners of numbering the faces. On the other hand, the quantity $z_{\mu} 2^{\ell(\mu)}$ is relevant for numbering and rooting the connected components. We use the double counting method and conclude the statement.

\section{The top-degree part of structure constants}

In this section we introduce the normalized Jack characters $\mathrm{Ch}_{\pi}$ and their structure constants $g_{\pi, \sigma}^{\mu}$. We present the formula for the top-degree part of structure constants. This rest of the section is devoted to the proof of the aforementioned formula. Firstly, we present some basic computations leading to the exact formulas for the top-degree part of Jack characters. We present those formulas in terms of injective embeddings of partitions into Young diagrams. Secondly, we consider a particular class of collections of bipartite maps $P_{\pi, \sigma}^{\mu}$ which constitute a good candidate for the top-degree parts of the structure constants $g_{\pi, \sigma}^{\mu}$. Finally, we prove that those candidates for the top-degree part of structure constants $g_{\pi, \sigma}^{\mu}$ (see Proposition 4.14) are indeed them.

\subsection{Normalized Jack characters}

We define Jack characters $\mathrm{Ch}_{\pi}$ by a choice of the normalization of $\theta_{\pi}^{(\alpha)}$. We will use the normalization introduced by Dołęga and Féray [DF16] which offers some advantages over the original normalization of Lassalle. Therefore, with the right choice of the multiplicative constant, the unnormalized Jack character $\theta_{\lambda}^{(\alpha)}(\pi)$ from (5) becomes the normalized Jack character $\mathrm{Ch}_{\pi}^{(\alpha)}(\lambda)$, defined as follows.

Definition 4.1. For a given number $\alpha>0$ and a partition $\pi$, the normalized Jack character $\mathrm{Ch}_{\pi}^{(\alpha)}(\lambda)$ is defined by:

$$
\mathrm{Ch}_{\pi}^{(\alpha)}(\lambda):= \begin{cases}\frac{1}{\sqrt{\alpha}}^{|\pi|+\ell(\pi)}\left(\begin{array}{c}
|\lambda|-|\pi|+m_{1}(\pi) \\
m_{1}(\pi)
\end{array}\right) z_{\pi} \theta_{\pi \cup 1|\lambda|-|\pi|}^{(\alpha)}(\lambda) & \text { if }|\lambda| \geqslant|\pi|, \\
0 & \text { if }|\lambda|<|\pi|,\end{cases}
$$


where $z_{\pi}$ is the standard numerical factor, and $\cup$ denotes concatenation of two partitions, see Section 2.1. The choice of an empty partition $\pi=\emptyset$ is acceptable; in this case $\mathrm{Ch}_{\emptyset}^{(\alpha)}(\lambda)=1$ [Śn19, Theorem 10.3].

\subsection{Structure constants}

Jack characters functions $\mathrm{Ch}_{\mu}$ span linearly, so-called, algebra of $\alpha$-polynomial functions [KO94, DF16]. Structure constants $g_{\pi, \sigma}^{\mu}$ of Jack characters are defined by expansion of the pointwise product of two Jack characters in the basis of Jack characters:

$$
\mathrm{Ch}_{\pi} \cdot \mathrm{Ch}_{\sigma}=\sum_{\mu} g_{\pi, \sigma}^{\mu}(\delta) \mathrm{Ch}_{\mu} .
$$

Explicit motivation for studying such quantities comes from a special choice of the deformation parameter $\alpha=1$, when Jack polynomials coincide with Schur polynomials. In this case, Frobenius duality ensures that the structure constants coincide with the connection coefficients for the symmetric groups [IK99].

Dołęga and Féray proved [DF16, Theorem 1.4] that each structure constant $g_{\pi, \sigma}^{\mu}$ is a polynomial in the variable $\delta:=\sqrt{\alpha}-\frac{1}{\sqrt{\alpha}}$ of degree bounded as follows:

$$
\operatorname{deg}_{\delta} g_{\pi, \sigma}^{\mu} \leqslant \min _{i=1,2,3}\left(n_{i}(\pi)+n_{i}(\sigma)-n_{i}(\mu)\right)
$$

where

$$
\begin{aligned}
& n_{1}(\pi)=|\pi|+\ell(\pi), \\
& n_{2}(\pi)=|\pi|-\ell(\pi), \\
& n_{3}(\pi)=|\pi|-\ell(\pi)+m_{1}(\pi) .
\end{aligned}
$$

For example, we have

$$
\begin{aligned}
\mathrm{Ch}_{3} \mathrm{Ch}_{2}= & 6 \delta \mathrm{Ch}_{3}+\mathrm{Ch}_{3,2}+6 \mathrm{Ch}_{2,1}+6 \mathrm{Ch}_{4} \\
\mathrm{Ch}_{3} \mathrm{Ch}_{3}= & \left(6 \delta^{2}+3\right) \mathrm{Ch}_{3}+9 \delta \mathrm{Ch}_{2,1}+18 \delta \mathrm{Ch}_{4}+3 \mathrm{Ch}_{1,1,1}+ \\
& +9 \mathrm{Ch}_{3,1}+9 \mathrm{Ch}_{2,2}+9 \mathrm{Ch}_{5}+\mathrm{Ch}_{3,3} .
\end{aligned}
$$

The numerical computations, such as the ones above, suggest that the structure constants of Jack characters might have some algebraic and combinatorial structure, which was proposed by Śniady in the following conjecture [Śn19].

Conjecture 4.2 (Structure constants of Jack characters). For any partitions $\pi, \sigma, \mu$, the corresponding structure constant

$$
g_{\pi, \sigma}^{\mu}(\delta) \in \mathbb{Q}[\delta]
$$

is a polynomial with non-negative integer coefficients. 


\subsection{The deformation parameters.}

In order to avoid dealing with the square root of the variable $\alpha$ we introduce an indeterminate $A$ such that $A^{2}:=\alpha$. Jack characters are usually defined in terms of the deformation parameter $\alpha$. After the substitution $\alpha:=A^{2}$, each Jack character becomes a function of $A$. In order to keep the notation light, we will make this dependence implicit and we will simply write $\mathrm{Ch}_{\pi}(\lambda)$.

The algebra of Laurent polynomials in the indeterminate $A$ variable will be denoted by $\mathbb{Q}\left[A, A^{-1}\right]$. For an integer $d$ we will say that a Laurent polynomial

$$
f=\sum_{k \in \mathbb{Z}} f_{k} A^{k} \in \mathbb{Q}\left[A, A^{-1}\right]
$$

is of degree at most $d$ if $f_{k}=0$ holds for each integer $k>d$.

A special role will be played by the quantity

$$
\delta:=A-\frac{1}{A} \in \mathbb{Q}\left[A, A^{-1}\right] .
$$

\subsection{The top-degree part of structure constants.}

We present an explicit formula for the top-degree part of structure constants of Jack characters.

Let us recall that we present an oriented map as a graph on the plane with a fixed cyclic order of outgoing edges together with a choice of the root, see Figure 8. Notice that with such presentation edges of the graph might cross. By convention we fixed the counter-clockwise orientation around vertices or, equivalently, the clockwise orientation of the page, see Figure 8. Similarly, we will present a $\mu$-collections of maps.

Let us recall that $\widetilde{M}_{\pi, \sigma}^{\bullet ; \mu}$ denotes the set of all $\mu$-lists of bipartite rooted and oriented maps which satisfy

$$
\Lambda_{\mathcal{W}}(M)=\pi \quad \text { and } \quad \Lambda_{\mathcal{B}}(M)=\sigma,
$$

see Figure 9.

Theorem 4.3. For any triple of partitions $\pi, \sigma, \mu$, the corresponding polynomial $g_{\pi, \sigma}^{\mu}(\delta)$ achieves the upper bounds on the degree

$$
d(\pi, \sigma ; \mu):=(|\pi|-\ell(\pi))+(|\sigma|-\ell(\sigma))-(|\mu|-\ell(\mu))
$$

if and only if $|\mu| \geqslant|\pi|,|\sigma|$, and $m_{1}(\pi)+m_{1}(\sigma) \geqslant m_{1}(\mu)$, and partitions $\pi \cup 1^{|\mu|-|\pi|}$ and $\sigma \cup 1^{|\mu|-|\sigma|}$ are joint sub-partitions of $\mu$ according to Definition 1.3. For such partitions, the leading coefficient of $g_{\pi, \sigma}^{\mu}(\delta)$ is a positive integer expressed in the following way:

$$
\left[\delta^{d(\pi, \sigma ; \mu)}\right] g_{\pi, \sigma}^{\mu}=C(\pi, \sigma ; \mu) \cdot \frac{z_{\pi} z_{\sigma}}{z_{\mu}}\left|\widetilde{M}_{\pi \cup 1|\mu|-|\pi|, \sigma \cup 1|\mu|-|\sigma|}^{\bullet ; \mu}\right|
$$




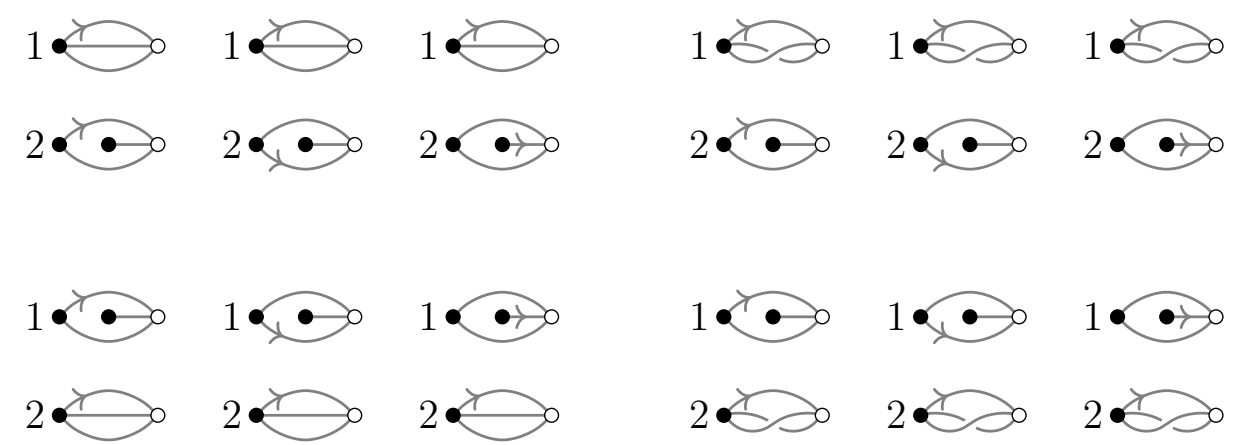

(a) All lists of maps in the set $\widetilde{M}_{\pi, \sigma \cup 1}^{\bullet ; \mu}$ for partitions $\pi=(3,3), \sigma=(3,2)$, and $\mu=(3,3)$. Those lists of maps consist of two connected components which are numbered by 1 and 2 , each has 3 edges. The vertex structure is given by $\pi$ and $\sigma$.

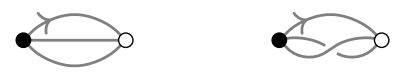

(b) Maps from the set $\widetilde{M}_{(3),(3)}^{\bullet ;(3)}$. Each of them can be rooted in the unique way.

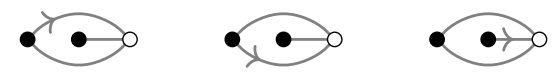

(c) Three rooted maps from the set $\widetilde{M}_{(3),(2,1)}^{\bullet ;}$. They are all the same as unrooted maps.

Figure 9 - There are twelve lists of maps in a set $\widetilde{M}_{\pi, \sigma \cup 1}^{\bullet ; \mu}$ for partitions $\pi=(3,3), \sigma=(3,2)$, and $\mu=(3,3)$, see Figure 9a. Each of them consists of a map from $\widetilde{M}_{(3),(3)}^{\bullet ;(3)}$ and $\widetilde{M}_{(3),(2,1)}^{\bullet ;(3)}$ presented on Figure 9b and Figure 9c respectively.

where

$$
\begin{aligned}
C(\pi, \sigma ; \mu)=\sum_{k=0}^{m_{1}(\mu)}\left(\begin{array}{c}
m_{1}(\mu) \\
k
\end{array}\right)\left(\begin{array}{c}
m_{1}(\pi)+|\mu|-|\pi|-m_{1}(\mu) \\
m_{1}(\pi)-k
\end{array}\right) . \\
\left.\qquad \begin{array}{c}
m_{1}(\sigma)+|\mu|-|\sigma|-m_{1}(\mu)+k \\
m_{1}(\sigma)-m_{1}(\mu)+k
\end{array}\right) .
\end{aligned}
$$

The remaining part of Section 4 is devoted to the proof of above theorem.

Example 4.4. Let us consider three partitions $\pi=(3,2), \sigma=(3,3)$, and $\mu=(3,3)$. In Figure 9 we have shown that $\widetilde{M}_{\pi, \sigma \cup 1}^{\bullet ; \mu}=12$. Using the theorem above, the $d(\pi, \sigma ; \mu)$ coefficient is equal to

$$
\left[\delta^{d(\pi, \sigma ; \mu)}\right] g_{\pi, \sigma}^{\mu}=\frac{6 \cdot 18}{18}\left(\begin{array}{l}
1 \\
0
\end{array}\right)\left(\begin{array}{l}
0 \\
0
\end{array}\right) 12=72
$$

\subsection{Embeddings of bicolored graphs}

A bicolored graph $G$ is a bipartite graph together with a choice of the colouring of its vertex set $\mathcal{V}$; we denote by $\mathcal{V}_{\bullet}$ and $\mathcal{V}_{\circ}$ respectively the sets of black and white vertices of $G$. 

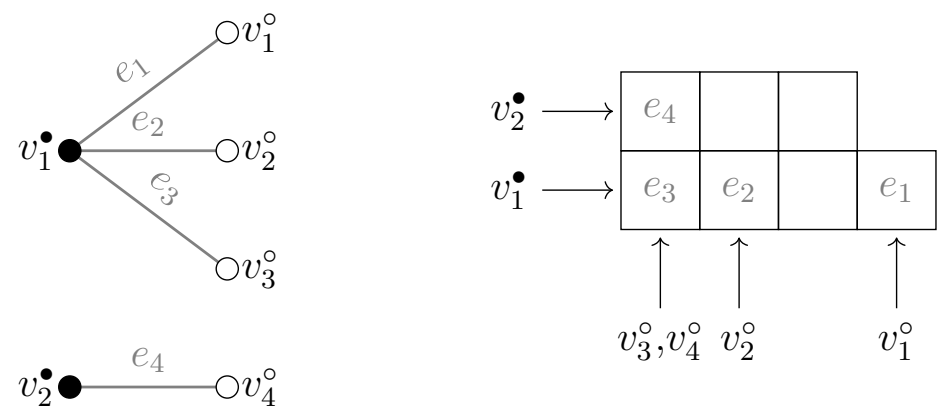

Figure 10 - The graph $G_{\pi}$ associated with the partition $\pi=(3,1)$. On the right, an example of its injective embedding into the Young diagram $\lambda=(4,3)$.

Definition 4.5. An injective embedding $F$ of a bicolored graph $G$ to a Young diagram $\lambda$ is a function which maps $\mathcal{V}_{\circ}$ to the set of columns of $\lambda$, maps $\mathcal{V} \bullet$ to the set of rows of $\lambda$, and maps injectively the set of edges $\mathcal{E}$ to the set of boxes of $\lambda$, see Figure 10. We also require that $F$ preserves the relation of incidence, i.e. each vertex $v \in \mathcal{V}$ should be mapped to a row or a column $F(v)$ which contains the box $F(e)$, for every edge $e \in \mathcal{E}$ incident to $v$. We denote by $N_{G}(\lambda)$ the number of such embeddings of $G$ into $\lambda$.

It is also useful to consider injective embeddings of a graph $G$ into a Young diagram $\lambda$, with the roles of black and white vertices reversed (i.e. black vertices are mapped into columns, white vertices into the rows). We refer to such embeddings as negative injective embeddings and denote the number of such embeddings as $\overline{N_{G}(\lambda)}$.

Definition 4.6. For any partition $\pi=\left(\pi_{1}, \ldots, \pi_{r}\right)$ we define the graph $G_{\pi}$ as the unique bicoloured graph consisting of $r$ black vertices of degrees $\pi_{1}, \ldots, \pi_{r}$ respectively and $|\pi|$ white vertices, each of degree one (see Figure 10). Similarly, we define $\overline{G_{\pi}}$ as the unique bicoloured graph consisting of $r$ white vertices of degrees $\pi_{1}, \ldots, \pi_{r}$ respectively and $|\pi|$ black vertices, each of degree one.

Remark 4.7. The number $N_{G_{\pi}}(\lambda)$ of injective embeddings of the graph $G_{\pi}$ into the Young diagram $\lambda$ is equal to the number $\overline{N_{\overline{G_{\pi}}}(\lambda)}$ of negative injective embeddings of the graph $\overline{G_{\pi}}$ into the Young diagram $\lambda$.

\subsection{Exact formulas for top-degree part of Jack characters}

Śniady proved [Śn19, Proposition 5.5] that each Jack character is a function on the set $\mathbb{Y}$ of Young diagrams

$$
\mathbb{Y} \ni \lambda \quad \longmapsto \quad \mathrm{Ch}_{\pi}(\lambda) \in \mathbb{Q}\left[A, A^{-1}\right]_{|\pi|-\ell(\pi)}
$$

with values in the set $\mathbb{Q}\left[A, A^{-1}\right]_{|\pi|-\ell(\pi)}$ of Laurent polynomials in the variable $A$ of degree at most $|\pi|-\ell(\pi)$. We denote by

$$
\left[A^{\mathrm{top}}\right] \mathrm{Ch}_{\pi}(\lambda):=\left[A^{|\pi|-\ell(\pi)}\right] \mathrm{Ch}_{\pi}(\lambda)
$$


the leading part of this Laurent polynomial. We shall express this quantity in terms of injective embeddings of $G_{\pi}$ into $\lambda$.

Proposition 4.8. For any Young diagram $\lambda \in \mathbb{Y}$ and partition $\pi$, we have that

$$
\left[A^{\text {top }}\right] \mathrm{Ch}_{\pi}(\lambda)=N_{G_{\pi}}(\lambda)
$$

That is, the leading part of $\mathrm{Ch}_{\pi}(\lambda)$ is equal to the number of injective embeddings of the graph $G_{\pi}$ into the Young diagram $\lambda$.

Example 4.9. Let us consider the partition $\pi=(3,1)$ and the Young diagram $\lambda=\left(\lambda_{1}, \lambda_{2}\right)$. We have

$$
\begin{aligned}
{\left[A^{\mathrm{top}}\right] \mathrm{Ch}_{(3,1)}\left(\lambda_{1}, \lambda_{2}\right)=N_{G_{\pi}}(\lambda)=\lambda_{1}{ }^{\underline{4}} } & +\lambda_{1}{ }^{\underline{3}} \cdot \lambda_{2}{ }^{\underline{1}} \\
& +\lambda_{1} \underline{\underline{1}} \cdot \lambda_{2}{ }^{\underline{3}}+\lambda_{2} \underline{\underline{4}},
\end{aligned}
$$

where $x^{k}:=x(x-1) \cdots(x-k+1)$ denotes the falling factorial. One of embeddings which contributes to $N_{G_{\pi}}(\lambda)$ is presented on Figure 10.

Before proving Proposition 4.8 we introduce the notion of $\alpha$-shifted symmetric functions (see more in [Las08, Section 2.2] or [AF17, Definition 2.2]) and present Jack characters in this context.

Definition 4.10. An $\alpha$-shifted symmetric function $F=\left(F_{N}\right)_{N \geqslant 1}$ is a sequence of polynomials $F_{N}$ such that

- for each $N \geqslant 1, F_{N}$ is a polynomial in $N$ variables $x_{1}, \ldots, x_{N}$ with coefficients in the field of rational functions $\mathbb{Q}(\alpha)$ in some indeterminate $\alpha$ that is symmetric in the variables

$$
\xi_{1}:=x_{1}-\frac{1}{\alpha}, \quad \xi_{2}:=x_{2}-\frac{2}{\alpha}, \ldots, \quad \xi_{N}:=x_{N}-\frac{N}{\alpha}
$$

- for each $N \geqslant 1, F_{N+1}\left(x_{1}, \ldots, x_{N}, 0\right)=F_{N}\left(x_{1}, \ldots, x_{N}\right)$ (the stability property),

- $\sup _{N \geqslant 1} \operatorname{deg}\left(F_{N}\right)<\infty$.

The degree of a shifted-symmetric function $F$ is defined as maximum of the degrees of the corresponding polynomials $F_{N}\left(x_{1}, \ldots, x_{N}\right)$. For an $\alpha$-shifted symmetric function $F$ and a Young diagram $\lambda=\left(\lambda_{1}, \ldots, \lambda_{r}\right)$, we introduce the notation

$$
F(\lambda):=F_{r}\left(\lambda_{1}, \ldots, \lambda_{r}\right) .
$$

Śniady and Féray gave some abstract characterizations of Jack characters [Śn15, Theorem 1.7, Theorem A.2]. We present the one given by Féray, which can be traced back to the earlier work of Knop and Sahi [KS96].

Theorem 4.11. [Śn15, Theorem A.2] Let $\pi$ be a partition and $A$ be a complex number such that $-\frac{1}{\alpha}=-\frac{1}{A^{2}}$ is not a positive integer. There exists a unique $\alpha$-shifted symmetric function $F$ such that: 
(J1) $F$ is a $\alpha$-shifted symmetric function of degree $|\pi|$, and its top-degree homogeneous part is equal to

$$
A^{|\pi|-\ell(\pi)} p_{\pi}\left(\lambda_{1}, \ldots, \lambda_{m}\right),
$$

where $p_{\pi}$ is the power-sum symmetric polynomial given by the formula

$$
p_{\pi}(\lambda)=\prod_{r} \sum_{i} \lambda_{i}^{\pi_{r}}
$$

(J2) $F(\lambda)=0$ holds for each Young diagram $\lambda$ such that $|\lambda|<|\pi|$ (the vanishing property).

Moreover, if $\alpha$ is a positive real number, the function $F=\left(F_{N}\right)_{N \geqslant 1}$ satisfies $\mathrm{Ch}_{\pi}(\lambda)=$ $F_{r}\left(\lambda_{1}, \ldots, \lambda_{r}\right)$ for each Young diagram $\lambda=\left(\lambda_{1}, \ldots, \lambda_{r}\right)$.

To keep notation short, we introduce the following symmetric function

$$
\widehat{p_{\pi}}(\lambda):=\underset{r}{*} \sum_{i} \lambda_{i}^{\frac{\pi_{r}}{i}}
$$

where

$$
\lambda_{i}^{\frac{l_{i}}{i}} * \lambda_{j}^{l_{j}}= \begin{cases}\lambda_{i}^{l_{i}+l_{j}} & \text { if } i=j, \\ \lambda_{i}^{\frac{l_{i}}{i}} \cdot \lambda_{j}^{\frac{l_{j}}{j}} & \text { otherwise }\end{cases}
$$

and

$$
\lambda^{\underline{l}}=\underbrace{\lambda \cdot(\lambda-1) \cdots(\lambda-l+1)}_{l \text { factors }} .
$$

Proof of Proposition 4.8. Observe that

$$
\widehat{p_{\pi}}(\lambda)=N_{G_{\pi}}(\lambda)
$$

We will show that

$$
\left[A^{\mathrm{top}}\right] \mathrm{Ch}_{\pi}(\lambda)=\widehat{p_{\pi}}(\lambda) \text {. }
$$

Let $F$ be an $\alpha$-shifted symmetric function associated to $\mathrm{Ch}_{\pi}$ by Theorem 4.11. Let us choose a sufficiently large integer $N$, e.g. $N>|\pi|$. Let us treat the coefficients of the polynomial $F_{N}$ as variables. The equality

$$
F_{N}(\lambda)=\mathrm{Ch}_{\pi}(\lambda) \in \mathbb{Q}\left[A, A^{-1}\right]_{|\pi|-\ell(\pi)},
$$

which holds for each $\lambda \in \mathbb{Y}$ such that $\ell(\lambda) \leqslant N$, becomes a system of equations with coefficients in $\mathbb{N}_{\geqslant 0}$. This system is invertible [KS96], hence we conclude that each coefficient of a polynomial $F_{N}$ is a linear combination of the quantities $\mathrm{Ch}_{\pi}(\lambda)$ over $\mathbb{Q}$. Therefore $F_{N}$ is a polynomial in $N$ variables with coefficients in $\mathbb{Q}\left[A, A^{-1}\right]_{|\pi|-\ell(\pi)}$.

Notice that formally we have equality for all $\alpha>0$. However, the rational function from $\mathbb{Q}(\alpha)$ is uniquely determined by its values for $\alpha \geqslant 0$. 
Since $F_{N}$ is a shifted-symmetric function with coefficients in the set $\mathbb{Q}\left[A, A^{-1}\right]_{|\pi|-\ell(\pi)}$, its $A$-top degree

$$
\left[A^{\text {top }}\right] F_{N}\left(\lambda_{1}, \ldots, \lambda_{N}\right):=\left[A^{|\pi|-\ell(\pi)}\right] F_{N}\left(\lambda_{1}, \ldots, \lambda_{N}\right)
$$

is a symmetric function in the variables $\lambda_{1}, \ldots, \lambda_{N}$. Indeed, for each permutation $\sigma$ of $[N]$ we have

$$
\begin{gathered}
{\left[A^{\mathrm{top}}\right] F_{N}\left(x_{1}, \ldots, x_{N}\right)=\left[A^{\mathrm{top}}\right] F_{N}\left(x_{1}-\frac{1}{A^{2}}, \ldots, x_{N}-\frac{N}{A^{2}}\right)=} \\
{\left[A^{\mathrm{top}}\right] F_{N}\left(x_{\sigma(1)}-\frac{\sigma(1)}{A^{2}}, \ldots, x_{\sigma(N)}-\frac{\sigma(N)}{A^{2}}\right)=} \\
{\left[A^{\mathrm{top}}\right] F_{N}\left(x_{\sigma(1)}, \ldots, x_{\sigma(N)}\right) .}
\end{gathered}
$$

Since $F_{N}$ is of a degree $|\pi|$, the polynomial $\left[A^{\text {top }}\right] F_{N}$ has the same bound of the degree. Observe that the homogeneous top-degree part of $\widehat{p_{\pi}}(\lambda)$ is equal to $p_{\pi}(\lambda)$ and so does the homogeneous top-degree part of $\left[A^{\mathrm{top}}\right] F_{N}$. Polynomials $\widehat{p_{\pi}}(\lambda)$ and $\left[A^{\text {top }}\right] F_{N}$ are both symmetric, hence

$$
\left[A^{\text {top }}\right] F_{N}-\widehat{p_{\pi}}
$$

is a symmetric polynomial in variables $\left(\lambda_{1}, \ldots, \lambda_{N}\right)$ of a degree at most $|\pi|-1$.

We use the following notation:

$$
\mathcal{Y}_{0}:=\left\{\left(\lambda_{1}, \ldots, \lambda_{N}\right) \in \mathbb{Z}^{N}: \lambda_{1} \geqslant \ldots \geqslant \lambda_{N} \geqslant 0 \text { and } \lambda_{1}+\ldots+\lambda_{N}<|\pi|\right\} .
$$

By the vanishing property we have

$$
\left[A^{\text {top }}\right] F_{N}(\lambda)=\left[A^{\text {top }}\right] \mathrm{Ch}_{\pi}(\lambda)=0
$$

for all elements $\lambda \in \mathcal{Y}_{0}$. Since there are no injective embeddings of $G_{\pi}$ into a Young diagram with the number of boxes smaller then the number of edges in $G_{\pi}$, we have

$$
\widehat{p_{\pi}}(\lambda)=N_{G_{\pi}}(\lambda)=0
$$

for all elements $\lambda \in \mathcal{Y}_{0}$. From that we deduce that $\mathcal{Y}_{0}$ is a set of zeros of the polynomial $\left[A^{\mathrm{top}}\right] F_{N}-\widehat{p_{\pi}}$. The appropriate set of zeros of a polynomial of sufficiently small degree determines the vanishing of the polynomial. Indeed, the symmetric polynomial

$$
W\left(x_{1}, \ldots, x_{N}\right):=\left(\left[A^{\mathrm{top}}\right] F_{N}-\widehat{p_{\pi}}\right)\left(x_{1}-1, \ldots, x_{N}-1\right),
$$

is of a degree at most $|\pi|-1$ and by the vanishing property, $W\left(x_{1}, \ldots, x_{N}\right)$ vanishes for all integers $x_{1}, \ldots, x_{N} \geqslant 1$ such that

$$
x_{1}+\ldots+x_{N}<|\pi|+N .
$$

Now, we can use the characterisation given by Śniady [Śn19, Lemma 7.6] to conclude that the polynomial $W\left(x_{1}, \ldots, x_{N}\right)$ vanishes. Hence we conclude that

$$
\left[A^{\text {top }}\right] F_{N}=\widehat{p_{\pi}}
$$

which finishes the proof. 


\subsection{Hands-shaking procedure}

Let

$$
\pi=\left(\pi_{1}, \ldots, \pi_{n}\right), \quad \sigma=\left(\sigma_{1}, \ldots, \sigma_{l}\right)
$$

be two partitions. We define a class of collections of maps by the following procedure:

Step 1. For each $i \in[n]$ we assign a white vertex with $\pi_{i}$ outgoing half-edges. We label this vertex by the number $i$ and we root it, i.e. we choose one of the outgoing half-edges and decorate it. Similarly, for each $j \in[l]$ we assign a black vertex with $\sigma_{j}$ outgoing half-edges and we root it.

Step 2. We match some of the half-edges going out from the white vertices with some of those going out from black vertices.

Step 3. We close each of the non-closed half-edges by a white or a black vertex so that the graph remains bipartite.

We call the procedure described above the "hands-shaking procedure". The name provenance could be explained as follows: there are white and black vertices with hands; the number of hands is given by the partitions $\pi$ and $\sigma$. They shake theirs hands in any way they like, but only black-white connections are allowed. On Figure 11 we present an example of applying this procedure.

Definition 4.12. For a given triple of partitions $\pi, \sigma, \mu$ we denote by $P_{\pi, \sigma}^{\mu}$ the set of all $\mu$-collections of maps (additionally with some vertices labelled and rooted) such that they may be obtained as an outcome of performing the above presented "hands-shaking procedure".

Each $\mu$-collection of maps $M \in P_{\pi, \sigma}^{\mu}$ can be obtained in the unique way as an outcome of the presented procedure. The uniqueness follows from the fact that the position of each edge from $M$ is uniquely determined by the labellings on the rooted vertices and the order of the outgoing half-edges.

Observation 4.13. For given partitions $\pi, \sigma, \mu$ : $|\pi|,|\sigma| \leqslant|\mu|$ we have

$$
\left|P_{\pi, \sigma}^{\mu}\right|=C(\pi, \sigma ; \mu) \cdot \frac{z_{\pi} z_{\sigma}}{z_{\mu}}\left|\widetilde{M}_{\pi \cup 1|\mu|-|\pi|, \sigma \cup 1|\mu|-|\sigma|}^{\bullet ; \mu}\right|
$$

where the coefficient

$$
\begin{aligned}
C(\pi, \sigma ; \mu)=\sum_{k=0}^{m_{1}(\mu)}\left(\begin{array}{c}
m_{1}(\mu) \\
k
\end{array}\right)\left(\begin{array}{c}
m_{1}(\pi)+|\mu|-|\pi|-m_{1}(\mu) \\
m_{1}(\pi)-k
\end{array}\right) . \\
\left.\qquad \begin{array}{c}
m_{1}(\sigma)+|\mu|-|\sigma|-m_{1}(\mu)+k \\
m_{1}(\sigma)-m_{1}(\mu)+k
\end{array}\right) .
\end{aligned}
$$

is relevant to the choice of degree-one vertices which are rooted. 


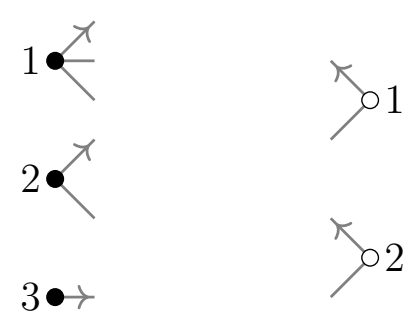

Step 1. Black and white vertices with outgoing half-edges of degrees $\left(\sigma_{i}\right)$ and $\left(\pi_{j}\right)$ respectively.

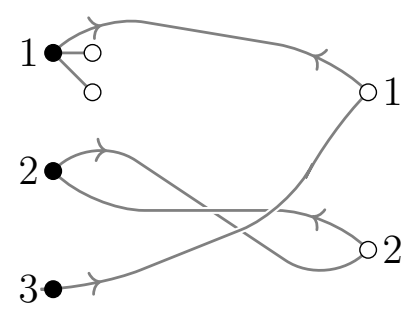

Step 3. The rest of outgoing half-edges is closed. The collection of maps is bipartite.

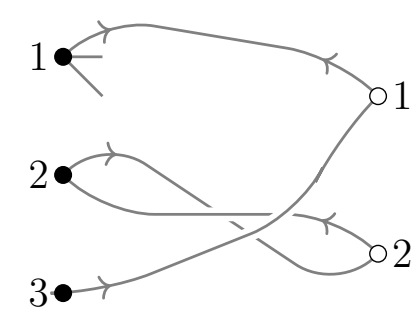

Step 2. Some of the outgoing half-edges were matched. The crossing of edges is not important.

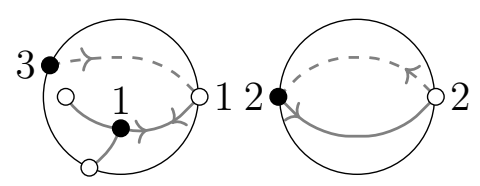

As an outcome we obtain the following collection of two maps drawn on a pair of spheres.

Figure 11 - The three steps of "hands-shaking procedure". As an output we obtain the $(4,2)$-collection of bipartite maps. Vertices are labelled and rooted as as the "handsshaking procedure" describes.

Proof. Observe that the elements of $P_{\pi, \sigma}^{\mu}$ are $\mu$-collection of bipartite orientable maps whose vertex set is given by

$$
\Lambda_{\mathcal{W}}(M)=\pi \cup 1^{|\mu|-|\pi|} \quad \text { and } \quad \Lambda_{\mathcal{B}}(M)=\sigma \cup 1^{|\mu|-|\sigma|} .
$$

Each such an element has the following labels and roots on the vertices and half-edges:

1. there are $n$ white vertices of degrees $\pi_{1}, \ldots, \pi_{n}$, each being labelled by a relevant natural number from $[n]$ and rooted, i.e. we choose one of the outgoing half-edges and decorate it by an arrow,

2. there are $l$ black vertices of degrees $\sigma_{1}, \ldots, \sigma_{l}$, each being labelled by a relevant natural number from $[l]$ and rooted.

Moreover, each connected component of an element from $P_{\pi, \sigma}^{\mu}$ has at least one decorated vertex. 
We use the double counting method as in Observation 3.10. For each $M \in P_{\pi, \sigma}^{\mu}$ we can root and number the connected components in $z_{\mu}$ ways.

Let us choose $M \in \widetilde{M}_{\pi \cup 1|\mu|-|\pi|, \sigma \cup 1|\mu|-|\sigma|}^{\bullet}$. The procedure of labelling and rooting the vertices is much more subtle. Firstly, we have to choose $m_{1}(\pi)$ white (respectively $m_{1}(\sigma)$ black) vertices and label them by adequate numbers. At the first sight, we could do this in

$$
\left(\begin{array}{c}
m_{1}(\pi)+|\mu|-|\pi| \\
m_{1}(\pi)
\end{array}\right)\left(\begin{array}{c}
m_{1}(\sigma)+|\mu|-|\sigma| \\
m_{1}(\sigma)
\end{array}\right) z_{\pi} z_{\sigma}
$$

ways (which is equal to $z_{\pi} z_{\sigma}$ if $\pi, \sigma, \mu$ are partitions of the same integer). However, in the definition of $P_{\pi, \sigma}^{\mu}$ we required to contain at least one labelled vertex from each connected component. This is trivially satisfied only if $m_{1}(\mu)=0$.

Observe, that connected components in $M$ which have more than one element, trivially contain at least one labelled vertex. Indeed, they contain at least one vertex of degree $d>2$, hence being labelled by some number. There are $m_{1}(\mu)$ one-element connected components in $M$. Denote the set of such connected components by $M_{1}$. Each connected component in $M_{1}$ consists of exactly two vertices: white and black. We can specify the number $k$ : $0 \leqslant k \leqslant m_{1}(\mu)$ of white vertices in $M_{1}$ which are labelled. For each integer $k$, we can choose $k$ white vertices in $M_{1}$ which are labelled on exactly $\left(\begin{array}{c}m_{1}(\mu) \\ k\end{array}\right)$ ways. Moreover, we can choose the remaining $m_{1}(\pi)-k$ white labelled vertices on exactly $\left(\begin{array}{c}m_{1}(\pi)+|\mu|-|\pi|-m_{1}(\mu) \\ m_{1}(\pi)-k\end{array}\right)$ ways. Indeed, the number of white vertices in $M \backslash M_{1}$ is equal to $m_{1}(\pi)+|\mu|-|\pi|-m_{1}(\mu)$. Observe, that in each connected component in $M_{1}$ which has no labelled white vertex, the black vertex has to be labelled. There are exactly $m_{1}(\mu)-k$ such vertices. Remaining $m_{1}(\sigma)-\left(m_{1}(\mu)-k\right)$ labelled black vertices might be chosen out of $m_{1}(\sigma)+|\mu|-|\sigma|-\left(m_{1}(\mu)-k\right)$ remaining degree-one black vertices, which contributes to the last factor in $(7)$. This analysis shows that the coefficient $C(\pi, \sigma ; \mu)$ is relevant to the number of ways of choosing labelled vertices in $M$. On the other hand, factors $z_{\pi} z_{\sigma}$ are relevant to the exact numbering among chosen black and white vertices. This finishes the proof of Observation 4.13.

\subsection{Proof of Theorem 4.3}

We prove that candidates $p_{\pi, \sigma}^{\mu}:=\left|P_{\pi, \sigma}^{\mu}\right|$ for top-degree part of structure constants $g_{\pi, \sigma}^{\mu}$ suit well for that role.

Proposition 4.14. For any Young diagram $\lambda \in \mathbb{Y}$, the following equality holds:

$$
\left[A^{\mathrm{top}}\right] \mathrm{Ch}_{\pi}(\lambda) \cdot\left[A^{\mathrm{top}}\right] \mathrm{Ch}_{\sigma}(\lambda)=\sum_{\mu} p_{\pi, \sigma}^{\mu}\left[A^{\mathrm{top}}\right] \mathrm{Ch}_{\mu}(\lambda) \text {. }
$$

Proof. According to Proposition 4.8, the two quantities

$$
\left[A^{\text {top }}\right] \mathrm{Ch}_{\sigma}(\lambda)=N_{G_{\sigma}}(\lambda) \quad \text { and } \quad\left[A^{\text {top }}\right] \mathrm{Ch}_{\mu}(\lambda)=N_{G_{\mu}}(\lambda)
$$

can be represented equivalently by the number of injective embeddings of $G_{\sigma}$ and $G_{\mu}$ into $\lambda$. Similarly,

$$
\left[A^{\text {top }}\right] \mathrm{Ch}_{\pi}(\lambda)=\overline{N_{\overline{G_{\pi}}}(\lambda)}
$$



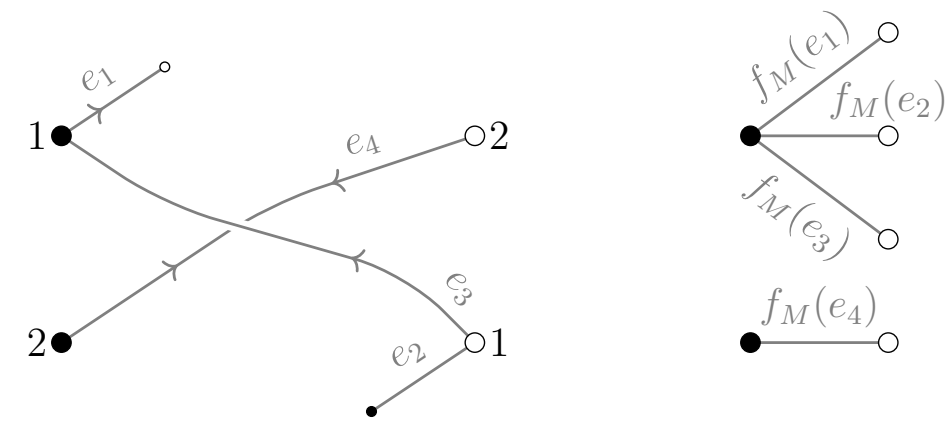

Figure 12 - Example of a collection of maps $M \in P_{(2,1),(2,1)}^{(3,1)}$ and an example of a bijection $f_{M}^{\mu}$ between the edges in $M$ and the edges of the graph $G_{(3,1)}$.

is equal to the number of negative injective embeddings of $\overline{G_{\pi}}$ into $\lambda$ (see Remark 4.7).

For each $M \in P_{\pi, \sigma}^{\mu}$ we choose some bijection $f_{M}^{\mu}$ between the edges of $M$ and the edges of the graph $G_{\mu}$ (see Definition 4.6), which preserves the connected components, see Figure 12.

We shall construct a bijection between:

- a pair $\left(\overline{N_{\overline{G_{\pi}}}(\lambda)}, N_{G_{\sigma}}(\lambda)\right)$ consisting of negative injective embeddings and injective embeddings of $G_{\sigma}$ and $\overline{G_{\pi}}$ into $\lambda$ respectively;

- a pair $\left(P_{\pi, \sigma}^{\mu}, N_{G_{\mu}}(\lambda)\right)$ consisting of collections of maps from the class $P_{\pi, \sigma}^{\mu}$ and injective embeddings of $G_{\mu}$ into $\lambda$.

The existence of such a bijection implies the statement of Proposition 4.14. We proceed analogously as in the "hands-shaking procedure" described in Section 4.7.

For each $i \in[n]$ we assign a white vertex with $\pi_{i}$ outgoing half-edges. We label this vertex by a number $i$ and root it, $i$.e. we choose one of outgoing half-edges and label it. We can choose a bijection between such half-edges and the edges in $\overline{G_{\pi}}$ which preserves the connected components. Similarly, for each $j \in[l]$ we assign a black vertex with $\sigma_{j}$ outgoing half-edges and we root it. Then we choose a bijection between such half-edges and the edges in $G_{\sigma}$ which preserves the connected components.

A reverse injective embedding of $\overline{G_{\pi}}$ and an injective embedding of $G_{\sigma}$ into $\lambda$ transfer into an injective embedding of above described half-edges going out from labelled and rooted black and white vertices.

We use the procedure described in Section 4.7 to connect in the unique way those outgoing half-edges which are embedded in the same box of Young diagram $\lambda$. We close each of non-closed half-edges by a white or a black vertex so that the graph remains bipartite.

In that way we obtain a list of maps $M \in P_{\pi, \sigma}^{\mu}$ injectively embedded into the Young diagram $\lambda$. Observe that all edges from any given connected component of $M$ are embed- 
ded into the boxes of $\lambda$ which are in the same row. Using the bijection $f_{M}^{\mu}$ between the edges of $M$ and the edges of $G_{\mu}$, we obtain the injective embedding of $G_{\mu}$ into $\lambda$.

The above procedure is reversible. Indeed, for a given collection of maps $M \in P_{\pi, \sigma}^{\mu}$ and an injective embedding of $G_{\mu}$ into diagram $\lambda$, we can easily construct the injective embedding of the edges of $M$ into the diagram $\lambda$, for which all edges from any given connected component of $M$ are embedded to the boxes from the same row. From such an object we can recover the elements from $N_{G_{\sigma}}(\lambda)$ and $\overline{N_{\overline{G_{\pi}}}(\lambda)}$.

With Proposition 4.14 in hand, we are ready to present the proof of Theorem 4.3.

Proof of Theorem 4.3. The upper bound of a degree for polynomials $g_{\pi, \sigma}^{\mu}(\delta)$ is given in (6). Since $\delta=A-\frac{1}{A}$, we have the following estimation

$$
\operatorname{deg}_{A} g_{\pi, \sigma}^{\mu}=\operatorname{deg}_{\delta} g_{\pi, \sigma}^{\mu} \leqslant d(\pi, \sigma ; \mu) .
$$

Let us fix a Young diagram $\lambda$. Recall that the evaluation of $\mathrm{Ch}_{\pi}$ on any Young diagram $\lambda$ is a Laurent polynomial in $\mathbb{Q}\left[A, A^{-1}\right]$ of a degree at most $n_{2}(\pi):=|\pi|-\ell(\pi)$. We investigate the $n_{2}(\pi)+n_{2}(\sigma)$ degree part of the pointwise product of two Jack characters, namely

$$
\left[A^{n_{2}(\pi)+n_{2}(\sigma)}\right] \mathrm{Ch}_{\pi}(\lambda) \cdot \mathrm{Ch}_{\sigma}(\lambda)=\left[A^{n_{2}(\pi)+n_{2}(\sigma)}\right] \sum_{\mu} g_{\pi, \sigma}^{\mu} \mathrm{Ch}_{\mu}(\lambda) .
$$

By the estimations on the upper bounds of the $A$-degrees of Laurent polynomials $\mathrm{Ch}_{\mu}(\lambda)$ and $g_{\pi, \sigma}^{\mu}$ we have

$$
\left[A^{\mathrm{top}}\right] \mathrm{Ch}_{\pi}(\lambda) \cdot\left[A^{\mathrm{top}}\right] \mathrm{Ch}_{\sigma}(\lambda)=\sum_{\mu}\left[A^{d(\pi, \sigma ; \mu)}\right] g_{\pi, \sigma}^{\mu}\left[A^{\mathrm{top}}\right] \mathrm{Ch}_{\mu}(\lambda) .
$$

We compare the above equation with Proposition 4.14 and we get

$$
\sum_{\mu}\left[A^{d(\pi, \sigma ; \mu)}\right] g_{\pi, \sigma}^{\mu}\left[A^{\text {top }}\right] \mathrm{Ch}_{\mu}(\lambda)=\sum_{\mu} p_{\pi, \sigma}^{\mu}\left[A^{\text {top }}\right] \mathrm{Ch}_{\mu}(\lambda)
$$

Recall that $\left[A^{\text {top }}\right] \mathrm{Ch}_{\mu}(\lambda)=\widehat{p_{\mu}}(\lambda)$. We have

$$
\sum_{\mu}\left[A^{d(\pi, \sigma ; \mu)}\right] g_{\pi, \sigma}^{\mu} \widehat{p_{\mu}}(\lambda)=\sum_{\mu} p_{\pi, \sigma}^{\mu} \widehat{p_{\mu}}(\lambda),
$$

The function $\widehat{p_{\mu}}(\lambda)$ is symmetric and its homogeneous top-degree part coincides with the power-sum symmetric polynomial $p_{\mu}$. This equality together with the fact that powersum symmetric functions form a basis of symmetric functions allows us to deduce that functions $\widehat{p_{\mu}}(\lambda)$ form also such a basis. We may look at (9) as an equality of symmetric functions. Since the basis determines its coefficients in the unique way, we conclude that

$$
\left[A^{d(\pi, \sigma ; \mu)}\right] g_{\pi, \sigma}^{\mu}=p_{\pi, \sigma}^{\mu}
$$

The $d(\pi, \sigma ; \mu)$-degree coefficients in variable $A$ and $\delta$ of $g_{\pi, \sigma}^{\mu}$ are equal. We conclude

$$
\left[\delta^{(\pi, \sigma ; \mu)}\right] g_{\pi, \sigma}^{\mu}=p_{\pi, \sigma}^{\mu} .
$$

Observation 4.13 finishes the enumerative part of the proof. In Observation 4.16 and Remark 4.17 we presents the necessary and sufficient condition for achieving the upper bound by polynomials $g_{\pi, \sigma}^{\mu}(\delta)$, which finishes the proof. 


\subsection{Necessary and sufficient conditions for achieving the upper bound}

We shall finish this section with a discussion when the upper bound for the top degree part of $g_{\pi, \sigma}^{\mu}$ presented in Theorem 4.3 is achieved. As we showed, it is enumerated by $\mu$-collections of maps with some vertices rooted which may be obtained as an outcome of performing the above presented "hands-shaking procedure". Therefore, we analyze, when the set of such collections $P_{\pi, \sigma}^{\mu}$ is non-empty.

Observation 4.15. Consider partitions $\pi, \sigma, \mu$. The following conditions are necessary for the set $P_{\pi, \sigma}^{\mu}$ being non-empty:

1. $|\pi|,|\sigma| \leqslant|\mu| \leqslant|\pi|+|\sigma|$,

2. both partitions $\pi \cup 1^{|\mu|-|\pi|}$ and $\sigma \cup 1^{|\mu|-|\sigma|}$ are sub-partitions of $\mu$,

3. $m_{1}(\pi)+m_{1}(\sigma) \geqslant m_{1}(\mu)$.

Proof. Observe that by performing "hands-shaking procedure", in which we obtain a $\mu$ collection of map, the vertex set is given by

$$
\Lambda_{\mathcal{W}}(M)=\pi \cup 1^{|\mu|-|\pi|} \quad \text { and } \quad \Lambda_{\mathcal{B}}(M)=\sigma \cup 1^{|\mu|-|\sigma|} .
$$

The first inequality in the first condition follows immediately. Moreover the number of edges in $P_{\pi, \sigma}^{\mu}$ cannot exceed $|\pi|+|\sigma|$, hence the second inequality in the first condition holds. Partitions describing white or black vertices distributions are sub-partitions of a partition describing distribution of connected components. Hence the second condition has to be satisfied. In the definition of $P_{\pi, \sigma}^{\mu}$ we required to contain at least one labelled vertex from each connected component. Hence the third condition has to be satisfied.

It is easy to see that conditions presented in Observation 4.15 are not sufficient for $P_{\pi, \sigma}^{\mu}$ being a non-empty set. Indeed, consider partitions $\pi=\sigma=\left(1^{n}\right)$, and the partition $\mu=(n)$. In such a situation, $P_{\pi, \sigma}^{\mu}$ consists of connected maps with all vertices of degree one, which is an empty set for $n>2$. The sufficient conditions are presented below.

Observation 4.16. Consider partitions $\pi, \sigma, \mu$ which satisfy $|\pi|,|\sigma| \leqslant|\mu|$ and $m_{1}(\pi)+$ $m_{1}(\sigma) \geqslant m_{1}(\mu)$. The set $P_{\pi, \sigma}^{\mu}$ is non-empty iff the partitions $\pi \cup 1^{|\mu|-|\pi|}$ and $\sigma \cup 1^{|\mu|-|\sigma|}$ are joint sub-partitions of $\mu$.

Proof. Consider firstly the case when $\mu=(n)$. We shall show that the set $P_{\pi, \sigma}^{(n)}$ is nonempty iff the partitions $\pi \cup 1^{|\mu|-|\pi|}$ and $\sigma \cup 1^{|\mu|-|\sigma|}$ are joint sub-partitions of $(n)$. This is equivalent to the following inequality:

$$
\ell\left(\pi \cup 1^{n-|\pi|}\right)+\ell\left(\sigma \cup 1^{n-|\sigma|}\right) \leqslant n+1 .
$$

We shall prove the "if and only if" statement inductively. Notice that for $n=1, m_{1}(\mu)=1$ and since we assumed $m_{1}(\pi)+m_{1}(\sigma) \geqslant m_{1}(\mu)$, one of the initial partitions $\pi$ or $\sigma$ is non-empty. Observe that under this assumption $P_{\pi, \sigma}^{(1)}$ is non-empty, and (10) is trivially satisfied. Suppose $n>1$. Without loss of generality, we may assume that $|\pi|=|\sigma|=|\mu|$. 
If $m_{1}(\sigma)=m_{1}(\pi)=0$, the condition (10) is satisfied. On the other hand, one can construct a map in $P_{\pi, \sigma}^{(n)}$. Indeed, in Step 2. of the "hands-shaking procedure", we may connect iteratively not-connected before edges. Since all vertices are of degree at least two, in some moment all vertices become connected. Other connections as well as labelling and rooting vertices are arbitrary. Suppose now that $m_{1}(\pi) \neq 0$ and choose the degree one part $\pi_{v}$. In order to construct a map in $P_{\pi, \sigma}^{(n)}$, we shall connect the vertex $\pi_{v}$ with the white vertex of degree at least two. Choose such a vertex $\sigma_{v}$. Consider two partitions: $\tilde{\pi}$ obtained from $\pi$ by removing the part $\pi_{v}$ and $\widetilde{\sigma}$ obtained from $\sigma$ by replacing the part $\sigma_{v}$ by $\sigma_{v}-1$. Partitions $\tilde{\pi}, \tilde{\sigma} \vdash n-1$ satisfies

$$
\ell(\tilde{\pi})+\ell(\widetilde{\sigma})<n,
$$

hence the problem of non-emptiness of $P_{\pi, \sigma}^{(n)}$ reduces to the problem of non-emptiness of $P_{\widetilde{\pi}, \widetilde{\sigma}}^{(n-1)}$, which ends the inductive step.

Consider the general case, when $\mu$ is arbitrary. Firstly, suppose that partitions $\pi \cup$ $1^{|\mu|-|\pi|}$ and $\sigma \cup 1^{|\mu|-|\sigma|}$ are joint sub-partitions of $\mu$. This means that they might be presented as the concatenations:

$$
\begin{aligned}
& \pi \cup 1^{|\mu|-|\pi|}=\pi^{1} \sqcup \cdots \sqcup \pi^{\ell(\mu)}, \\
& \sigma \cup 1^{|\mu|-|\sigma|}=\sigma^{1} \cdots \sqcup \sigma^{\ell(\mu)},
\end{aligned}
$$

which satisfy $\left|\pi^{i}\right|=\left|\sigma^{i}\right|=\left|\mu_{i}\right|$ and

$$
\ell\left(\pi^{i}\right)+\ell\left(\sigma^{i}\right) \leqslant\left|\mu_{i}\right|+1,
$$

for all indices $i \in \ell(\mu)$. Moreover, assuming $m_{1}(\pi)+m_{1}(\sigma) \geqslant m_{1}(\mu)$, in any pair $\pi^{i}$ or $\sigma^{i}$ of the division there might be a part from original partition $\pi$ or $\sigma$. Hence the condition $m_{1}\left(\pi^{i}\right)+m_{1}\left(\sigma^{i}\right) \geqslant m_{1}\left(\mu_{i}\right)$ is satisfied for any $i \in \ell(\mu)$. Furthermore, assuming that (11) is satisfied, for each pair $\pi^{i}, \sigma^{i}$, we construct the map from $P_{\pi^{i}, \sigma^{i}}^{\mu_{i}}$ according to the previous discussion. In such a way, we constructed the $\mu$-collection of maps from $P_{\pi, \sigma}^{\mu}$.

Secondly, we show that presented constraints are also necessary for $P_{\pi, \sigma}^{\mu}$ being nonempty set. Suppose that $P_{\pi, \sigma}^{\mu}$ is a non-empty set. According to Observation $4.15|\pi|,|\sigma| \leqslant$ $|\mu|$ and $m_{1}(\pi)+m_{1}(\sigma) \geqslant m_{1}(\mu)$ has to be satisfied. We shall show that (11) is also satisfied. Indeed, consider arbitrary $\mu$-collection of maps from $P_{\pi, \sigma}^{\mu}$. It imposes the division of partitions $\pi$ and $\sigma$ into $\pi^{i}$ and $\sigma^{i}$ according to the division into connected components. Each map from the $\mu$-collection satisfies (11) according to the previous discussion.

Remark 4.17. Notice that if $\mu, \pi, \sigma \vdash n$ are partitions of the same number, the set $P_{\pi, \sigma}^{\mu}$ is non-empty if $\pi$ and $\sigma$ are joint sub-partitions of $\mu$.

\section{Measures of non-orientability and non-bipartiteness}

\subsection{The $b$-Conjecture.}

Equations (1) and (2) define two families of coefficients $\left(c_{\pi, \sigma}^{\lambda}\right)$ and $\left(h_{\pi, \sigma}^{\lambda}\right)$. Goulden and Jackson [GJ96] discussed some specialisations of the family $\left(c_{\pi, \sigma}^{\lambda}\right)$ and hypothetical 
combinatorial interpretations of the polynomials $c_{\pi, \sigma}^{\lambda}$ in terms of matchings known as the Matchings-Jack Conjecture, see Section 1.5. In the same paper they observed that specializations of $h_{\pi, \sigma}^{\lambda}(\beta)$ for $\beta=0,1$ may be expressed in terms of rooted maps, namely

$$
\begin{aligned}
& h_{\pi, \sigma}^{\lambda}(0)=\mid\left\{M \in M_{\pi, \sigma}^{\lambda}: M \text { is orientable }\right\} \mid, \\
& h_{\pi, \sigma}^{\lambda}(1)=\left|\left\{M \in M_{\pi, \sigma}^{\lambda}\right\}\right| .
\end{aligned}
$$

Based on this observation Goulden and Jackson conjectured that the family $\left(h_{\pi, \sigma}^{\lambda}\right)$ of polynomials may have a combinatorial interpretation. The conjecture is known as the b-Conjecture.

Conjecture 5.1 (b-Conjecture). For any partitions $\pi, \sigma, \lambda \vdash n$ the quantity $h_{\pi, \sigma}^{\lambda}$ can be expressed as

$$
h_{\pi, \sigma}^{\lambda}(\beta)=\sum_{M \in M_{\pi, \sigma}^{\lambda}} \beta^{\eta(M)}
$$

where $\eta: M_{\pi, \sigma}^{\lambda} \longrightarrow \mathbb{N}_{0}$ is some hypothetical combinatorial statistic such that $\eta(M)=0$ if and only if $M$ is orientable.

\subsection{Root-deletion procedure and a measure of non-orientability.}

The statistic $\eta$ from the $b$-Conjecture should be a marker of non-orientability of maps. We shall present the definition of the measure of non-orientability introduced by La Croix [La 09, Definition 4.1], which seems to be a good candidate for the hypothetical statistic conjectured by Goulden and Jackson. We adapt the statistic given by La Croix to the case of lists of maps.

Definition 5.2 (Root-deletion procedure). Denote by $e$ the root edge of the map $M$. By deleting $e$ from $M$ we create either a new map, or two new maps. We give the canonical procedure of rooting it or them. Rooting a map is equivalent to choosing an oriented corner, see Figure 13. We denote such an oriented corner as the root corner. Denote by $c$ the root corner of $M$.

Suppose that $M \backslash e$ is connected. Observe that $c$ is contained in the unique oriented corner of $M \backslash e$, we define such an oriented corner as the root of $M \backslash e$.

Suppose that $M \backslash e$ has two connected components. One of them can be rooted as above. Observe that the first corner in the root face of $M$ following $c$ is contained in a unique oriented corner of the second component of $M \backslash e$, see Figure 13. We define this oriented corner as the root of this component.

Remark 5.3. The Root-deletion procedure is defined for all maps, not necessary bipartite. In particular, we do not require that the root vertex is black.

We classify the root edges of maps. Let $f$ be the number of faces of a map $M$ with the root vertex $e$;

1. $e$ is called a bridge if $M \backslash e$ is not connected, 


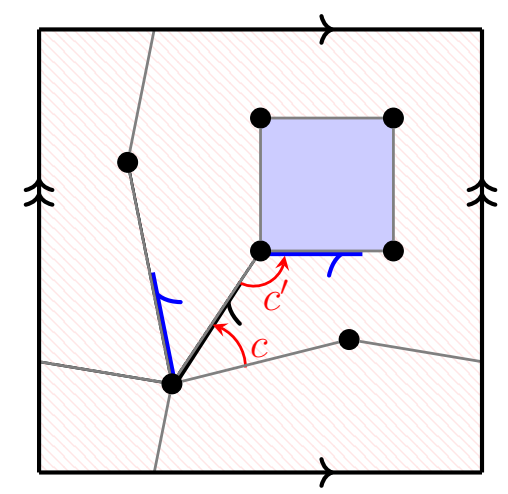

Figure 13 - The oriented corner $c$ (red arrow) equivalent to the root (the black arrow) of a map. The first corner in the root face of the map following $c$ is labelled by $c^{\prime}$ (red arrow). By deleting the root edge the map splits into two new maps. The oriented corners $c$ and $c^{\prime}$ are contained in two oriented corners of the new maps. They give the roots of those maps (the blue arrows).

2. otherwise $M \backslash e$ is connected and $e$ is called

- a border if the number of faces in $M \backslash e$ is equal to $f-1$,

- a twisted edge if the number of faces in $M \backslash e$ is equal to $f$,

- a handle if the number of faces in $M \backslash e$ is equal to $f+1$.

Remark 5.4. A leaf (i.e. an edge connecting a vertex of degree 1) is considered as a bridge. Definition 5.5. [La 09, Definition 4.1] A functional $\eta$ on the set of rooted maps is called a measure of non-orientability if it satisfies the following properties: follows.

1. If $M$ has no edges then $\eta(M)=0$.

2. Otherwise $M$ has the root edge $e$,

- $\eta(M)=\eta\left(M_{1}\right)+\eta\left(M_{2}\right)$ if $e$ is a bridge, while $M_{1}$ and $M_{2}$ are the connected components of $M \backslash e$,

- $\eta(M)=\eta(M \backslash e)$ if $e$ is a border,

- $\eta(M)=\eta(M \backslash e)+1$ if $e$ is a twisted edge,

- if $e$ is a handle, there exists a unique map $M^{\prime}$ with the root edge $e^{\prime}$ constructed by twisting the edge $e$ in $M$, in such a way that $e^{\prime}$ is a handle and the maps $M \backslash e, M^{\prime} \backslash e$ are equal. In this case we require that

$$
\left\{\eta(M), \eta\left(M^{\prime}\right)\right\}=\{\eta(M \backslash e), \eta(M \backslash e)+1\} .
$$

At most one of the maps $M, M^{\prime}$ is orientable. For such a map $M$ we require $\eta(M)=\eta(M \backslash e)$. 
Observe that the above definition introduces a whole family of measures of nonorientability $\eta$ and among of them there is no canonical measure of non-orientability.

Remark 5.6. For a given rooted map $M$

$$
\eta(M)=0 \text { if and only if } M \text { is orientable. }
$$

Indeed, removing twisted edges or handles during the root-deletion procedure are the only possibilities of increasing the recursively-defined statistic $\eta$. An orientable map does not have any twisted edges (a map with a twisted edge is embedded in a surface which contains the Möbius strip, hence is nonorientable). The recursive definition of $\eta$ guarantees that removing handles from an orientable map does not increase the statistic $\eta$. Hence for an orientable map $M$, we have $\eta(M)=0$. A reverse analysis or a simple induction on the number of edges provides the reverse implication.

Definition 5.7. Any measure of non-orientability $\eta$ from Definition 5.5 might be extended to the measure of non-orientability of rooted list of maps. For a rooted $\mu$-list of maps $M=M_{1}, \ldots, M_{k}$ we define its measure of non-orientability $\eta$ by

$$
\eta(M):=\eta\left(M_{1}\right)+\cdots+\eta\left(M_{k}\right) .
$$

\subsection{Unhandled and unicellular maps.}

Definition 5.8. The rooted map $M$ is called unhandled if by iteratively performing the root-deletion process (see Definition 5.2) it does not have any handles. The map $M$ is called unicellular if it has only one face.

From now on we fix one measure of non-orientability $\eta$ of the class of maps. Dołęga [Doł17, Section 4] showed that for such a measure $\eta$ the polynomial $H_{\eta}$ given by the sum

$$
\left(H_{\eta}\right)_{\pi, \sigma}^{\lambda}:=\sum_{M \in M_{\pi, \sigma}^{\lambda}} \beta^{\eta(M)}
$$

has degree at most equal to $n+1-\ell(\pi)-\ell(\sigma)$ and the leading coefficient is enumerated by unhandled unicellular maps. In particular, $\left(H_{\eta}\right)_{\pi, \sigma}^{\lambda}$ may achieve this bound of the degree only if $\lambda=(n)$. He also showed that the aforementioned leading coefficient is also enumerated by oriented maps with arbitrary face-type, namely

$$
\mid M \in M_{\pi, \sigma}^{\bullet}: M \text { is orientable }|=| M \in M_{\pi, \sigma}^{(n)}: M \text { is unhandled } \mid .
$$

In fact, there is an explicit bijection between those two families of maps. Dołega proved [Doł17, Theorem 1.4] that for the statistic $\eta$

$$
h_{\pi, \sigma}^{(n)}(\beta)=\sum_{M \in M_{\pi, \sigma}^{(n)}} \beta^{\eta(M)}
$$

holds true for $\beta \in\{-1,0,1\}$, furthermore $\eta(M)=n+1-\ell(\pi)-\ell(\sigma)$ if and only if $M$ is unhandled and unicellular. 
The result of Dołęga is easily transferable to the context of $\mu$-lists of maps. Let us choose the measures of non-orientability $\eta_{i}$ for $i \in[k], k=\ell(\mu)$, which form the measure $\eta$ as it is described in Definition 5.7.

Lemma 5.9. For the statistic $\eta$, the polynomial $\left(H_{\eta}\right)_{\pi, \sigma}^{\lambda ; \bullet}$ given by the sum

$$
\left(H_{\eta}\right)_{\pi, \sigma}^{\lambda ; \bullet}:=\sum_{\mu: \lambda \preceq \mu}\left(H_{\eta}\right)_{\pi, \sigma}^{\lambda ; \mu}
$$

where

$$
\left(H_{\eta}\right)_{\pi, \sigma}^{\lambda ; \mu}(\beta)=\sum_{M \in M_{\pi, \sigma}^{\lambda ; \mu}} \beta^{\eta(M)}
$$

is of degree at most $d(\pi, \sigma ; \lambda)$, see (4). Moreover, a $\mu$-list of maps $M$ contributes to the constant term if and only if $M$ is a list of orientable maps. The $\mu$-lists of maps $M$ contributes to the leading coefficient if and only if $M$ is a list of unicellular and unhandled maps, in particular $\mu=\lambda$.

Proof. Each $M=\left(M_{1}, \ldots, M_{k}\right) \in M_{\pi, \sigma}^{\lambda ; \mu}$ decompose into a list of maps $M_{i} \in M_{\pi_{|| \mu_{i}}, \sigma_{\mid \mu_{i}}}^{\lambda_{\mid \mu_{i}}}$ for some partitions $\pi_{\mid \mu_{i}}, \sigma_{\mid \mu_{i}}, \lambda_{\mid \mu_{i}} \vdash \mu_{i}$ satisfying

$$
\bigcup_{i=1}^{k} \pi_{\mid \mu_{i}}=\pi, \quad \bigcup_{i=1}^{k} \sigma_{\mid \mu_{i}}=\sigma, \quad \bigcup_{i=1}^{k} \lambda_{\mid \mu_{i}}=\lambda
$$

We denote by $\mathcal{P}_{\pi}^{\mu}$ the set of lists of partitions $\left(\pi_{\mid \mu_{1}}, \ldots, \pi_{\mid \mu_{k}}\right)$, where $\pi_{\mid \mu_{i}} \vdash \mu_{i}$ and

$$
\bigcup_{i=1}^{k} \pi_{\mid \mu_{i}}=\pi
$$

Observe, that (13) can be rewritten in such a way:

$$
\sum_{M \in M_{\pi, \sigma}^{\lambda ; \mu}} \beta^{\eta(M)}=\sum_{\substack{\left(\pi^{1}, \ldots, \pi^{k}\right) \in \mathcal{P}_{\mu}^{\mu} \\\left(\sigma^{1}, \ldots, \sigma^{k}\right) \in \mathcal{P}_{\mu}^{\mu} \\\left(\lambda^{1}, \ldots, \lambda^{k}\right) \in \mathcal{P}_{\lambda}^{\mu}}} \sum_{M \in M_{\pi^{i}, \sigma^{i}}^{\lambda^{i}}} \beta^{\eta_{i}(M)} .
$$

We use the result of Dołega applied to each right most side sum separately. Each such a sum has degree at most equal to $\mu_{i}+1-\ell\left(\pi^{i}\right)-\ell\left(\sigma^{i}\right)$ and the top-degree coefficient is enumerated by unhandled unicellular maps. Since

$$
n+\ell(\mu)-\ell(\pi)-\ell(\sigma)=\sum_{i=1}^{k}\left(\mu_{i}+1-\ell\left(\pi^{i}\right)-\ell\left(\sigma^{i}\right)\right),
$$

we conclude that (12) has degree at most equal to $d(\pi, \sigma ; \lambda)$ and the top-degree coefficient is enumerated by $\mu$-lists of unhandled unicellular maps. 
Corollary 5.10. For three given partitions $\pi, \sigma, \lambda \vdash n$ we have

$$
\mid M \in M_{\pi, \sigma}^{\bullet ; \mu}: M \text { is orientable }|=| M \in M_{\pi, \sigma}^{\mu ; \mu}: M \text { is unhandled } \mid .
$$

Proof. Fix a list $M \in M_{\pi, \sigma}^{\mu ; \mu}$ of unhandled and unicellular maps. For each connected component of $M$ we use the aforementioned bijection between such maps and oriented maps with arbitrary face-type given by Dołęga [Doł17, Corollary 3.10]. We get a $\mu$-list of orientable maps with arbitrary face type.

\subsection{Measure of non-bipartiteness for matchings.}

The hypothetical statistic $\mathrm{wt}_{\lambda}$ from the Matchings-Jack Conjecture should be a marker of non-bipartiteness for matchings. Naturally, matchings correspond to lists of maps, in particular bipartite matching to lists of oriented maps.

The naive thought how the statistic $\mathrm{wt}_{\lambda}$ should be defined is to adapt the measure of non-orientability introduced by La Croix by the correspondence between matchings and collections of maps given by Corollary 3.7. Regretfully, the measure introduced by La Croix is defined for lists of rooted maps, and it is not clear how to define it naturally for lists of maps with rooted faces in $M\left(\mathcal{G}_{\pi, \sigma}^{\lambda ; \mu}\right)$, and hence for matchings in $\mathcal{G}_{\pi, \sigma}^{\lambda ; \mu}$.

However, there is one special class of matchings, which may be identified with lists of rooted maps, namely $\mathcal{G}_{\pi, \sigma}^{\lambda ; \lambda}$. When the number of faces is equal to the number of connected components, numbering and rooting faces overlap with numbering and rooting components. For a fixed measure of non-orientability $\eta$ we define

$$
\begin{aligned}
\text { stat: } \mathcal{G}_{\pi, \sigma}^{\lambda ; \lambda} & \longrightarrow[d(\pi, \sigma ; \lambda)] \\
\delta & \longmapsto \operatorname{stat}(\delta):=\eta\left(M_{\delta}\right)
\end{aligned}
$$

For given partitions $\lambda, \pi, \sigma \vdash n$ we define the following polynomial

$$
\left(G_{\eta}\right)_{\pi, \sigma}^{\lambda ; \lambda}:=\sum_{\delta \in \mathcal{G}_{\pi, \sigma}^{\lambda ; \lambda}} \beta^{\operatorname{stat}(\delta)}
$$

Definition 5.11. We say that a matching $\delta \in \mathcal{G}_{\pi, \sigma}^{\lambda ; \lambda}$ is unhandled if the corresponding map $M_{\delta} \in M_{\pi, \sigma}^{\lambda ; \lambda}$ is so.

Lemma 5.12. For any triple of partitions $\pi, \sigma, \lambda \vdash n$ the corresponding polynomial $\left(G_{\eta}\right)_{\pi, \sigma}^{\lambda ; \lambda}$ is of degree at most $d(\pi, \sigma ; \lambda)$. Moreover, the matching $\delta$ contributes to the constant term if and only if $\delta$ is bipartite. The matching $\delta$ contributes to the leading coefficient if and only if $\delta$ is an unhandled matching.

Moreover, the top-degree coefficient may be enumerated in two different manners:

$$
\mid \delta \in \mathcal{G}_{\pi, \sigma}^{\lambda ; \lambda}: \delta \text { is unhandled }\left|=\sum_{\nu: \nu \preceq \lambda} \frac{z_{\lambda}}{z_{\nu}}\right| \delta \in \mathcal{G}_{\pi, \sigma}^{\nu ; \lambda}: \delta \text { is bipartite } \mid .
$$

Proof. Observe that for a fixed measure of non-orientability $\eta$, the polynomials $\left(G_{\eta}\right)_{\pi, \sigma}^{\lambda ; \lambda}$ and $\left(H_{\eta}\right)_{\pi, \sigma}^{\lambda ; \lambda}$ are equal. The first statement follows immediately from Lemma 5.9. The second statement is an easy conclusion of Corollary 5.10 and relation given in Observation 3.12. 


\section{Relations between the structure constants $g_{\pi, \sigma}^{\mu}$ and the con- nection coefficients $c_{\pi, \sigma}^{\mu}$}

Structure constants $g_{\pi, \sigma}^{\mu}$ and the connection coefficients $c_{\pi, \sigma}^{\mu}$ are closely related notions. In Section 4 the formula for the top-degree part of structure constants is presented, see Theorem 4.3. We discuss relations between both families of coefficients $g_{\pi, \sigma}^{\mu}$ and $c_{\pi, \sigma}^{\mu}$, and recover the formula for the top-degree part of connection coefficients, which is presented in Theorem 1.5.

It is worth mentioning that the coefficients $c_{\pi, \sigma}^{\mu}$ are indexed by three partitions of the same size, while the quantities $g_{\pi, \sigma}^{\mu}$ are indexed by triples of arbitrary partitions. Dołęga and Féray investigated the relationship between these two families of coefficients and showed [DF16, Equation (19)] that for $\mu, \pi, \sigma \vdash n$,

$$
c_{\pi, \sigma}^{\mu}=\sqrt{\alpha}^{d(\pi, \sigma ; \mu)} \frac{z_{\tilde{\mu}}}{z_{\tilde{\pi}} z_{\tilde{\sigma}}} \sum_{i=0}^{m_{1}(\pi)} g_{\tilde{\pi}, \tilde{\sigma}}^{\tilde{\mu} \cup 1^{i}} \cdot i !\left(\begin{array}{c}
n-|\tilde{\mu}| \\
i
\end{array}\right),
$$

where $\widetilde{\pi}$ is constructed from the partition $\pi$ by deleting all units.

Dołęga and Féray [DF16] proven the polynomiality and the bound on the degree of $g_{\pi, \sigma}^{\mu}$. Using (15) they deduced the polynomiality and the bound of the degree of connection coefficients $c_{\pi, \sigma}^{\mu}$. We establish other relations between those two families of coefficients.

Corollary 6.1. For three given partitions $\mu, \pi, \sigma \vdash n$, each of the polynomials $c_{\pi, \sigma}^{\mu}(\beta)$ and $g_{\tilde{\pi}, \tilde{\sigma}}^{\tilde{\alpha}}(\delta)$ is of degree at most $d(\pi, \sigma ; \mu)$, and their leading coefficients coincide up to a normalizing constant, namely

$$
\left[\beta^{d(\pi, \sigma ; \mu)}\right] c_{\pi, \sigma}^{\mu}=\frac{z_{\tilde{\mu}}}{z_{\tilde{\pi}} z_{\tilde{\sigma}}} \cdot\left[\delta^{d(\pi, \sigma ; \mu)}\right] g_{\tilde{\pi}, \tilde{\sigma}}^{\tilde{\mu}}
$$

Proof. Fix three partitions $\mu, \pi, \sigma \vdash n$. Observe that for each $i \geqslant 0$, the third estimation shown in (6) gives us

$$
\operatorname{deg}_{\delta} g_{\tilde{\pi}, \tilde{\sigma}}^{\tilde{\mu} \cup 1^{i}} \leqslant d(\pi, \sigma ; \mu)-i .
$$

Let us recall that $\delta=\sqrt{\alpha}-\frac{1}{\sqrt{\alpha}}$, hence the right-hand side of (15) is of $\sqrt{\alpha}$-degree at most equal to $2 d(\pi, \sigma ; \mu)$, and in the sum over $i$, the only contribution to the $2 d(\pi, \sigma ; \mu)$-degree coefficient comes from $g_{\tilde{\pi}, \tilde{\sigma}}^{\tilde{\tilde{\alpha}}}$. We have

$$
\left[\sqrt{\alpha}^{2 d(\pi, \sigma ; \mu)}\right]\left(\sqrt{\alpha}^{d(\pi, \sigma ; \mu)} \frac{z_{\tilde{\mu}}}{z_{\tilde{\pi}} z_{\tilde{\sigma}}} \sum_{i=0}^{m_{1}(\pi)} g_{\tilde{\pi}, \tilde{\sigma}}^{\tilde{\mu} \cup 1^{i}} \cdot i !\left(\begin{array}{c}
n-|\tilde{\mu}| \\
i
\end{array}\right)\right)=\frac{z_{\tilde{\mu}}}{z_{\tilde{\pi}} z_{\tilde{\sigma}}}\left[\delta^{d(\pi, \sigma ; \mu)}\right] g_{\tilde{\pi}, \tilde{\sigma}}^{\tilde{\mu}}
$$

Since $\beta=\alpha-1$, the $2 d(\pi, \sigma ; \mu)$-degree coefficient of $c_{\pi, \sigma}^{\mu}$ in variable $\sqrt{\alpha}$ coincides with $d(\pi, \sigma ; \mu)$-degree coefficient in variable $\beta$. Hence (15) finishes the proof.

Assuming Theorem 4.3 we are ready to prove the main result of this paper. The proof may seem intricate, it combines different facts which have been proven so far. 
Proof of Theorem 1.5. Fix partitions $\pi, \sigma, \lambda \vdash n$. We investigate the polynomial $c_{\pi, \sigma}^{\lambda}(\beta)$. By Corollary 6.1 we have

$$
\left[\beta^{d(\pi, \sigma ; \lambda)}\right] c_{\pi, \sigma}^{\lambda}=\frac{z_{\tilde{\lambda}}}{z_{\tilde{\pi}} z_{\tilde{\sigma}}} \cdot\left[\delta^{d(\pi, \sigma ; \lambda)}\right] g_{\tilde{\pi}, \tilde{\sigma}}^{\tilde{\lambda}}
$$

and by Theorem 4.3 we know that the polynomial $g_{\tilde{\pi}, \tilde{\sigma}}^{\tilde{\sigma}}$ achieves the $d(\pi, \sigma ; \lambda)$-degree part if and only if $|\tilde{\lambda}| \geqslant|\tilde{\pi}|,|\tilde{\sigma}|, \tilde{\pi} \cup 1^{|\tilde{\lambda}|-|\tilde{\pi}|} \preceq \tilde{\lambda}$, and $\tilde{\sigma} \cup 1^{|\tilde{\lambda}|-|\tilde{\sigma}|} \preceq \tilde{\lambda}$. Observe that this condition is equivalent to $\pi \preceq \lambda$ and $\sigma \preceq \lambda$. Hence the condition on partitions $\pi, \sigma, \lambda$ for achieving by $c_{\pi, \sigma}^{\lambda}$ the $d(\pi, \sigma ; \lambda)$-degree.

Thus, we have

$$
\left[\beta^{d(\pi, \sigma ; \lambda)}\right] c_{\pi, \sigma}^{\lambda} \stackrel{\text { Corollary } 6.1}{=} \frac{z_{\tilde{\lambda}}}{z_{\tilde{\pi}} z_{\tilde{\sigma}}} \cdot\left[\delta^{d(\pi, \sigma ; \lambda)}\right] g_{\tilde{\pi}, \tilde{\sigma}}^{\tilde{\lambda}} \stackrel{\text { Theorem }}{=} 4.3\left|\widetilde{M}_{\tilde{\pi}, \tilde{\sigma}}^{\bullet ; \tilde{\lambda}}\right| \cdot
$$

Since there is only one map $M_{1} \in \widetilde{M}_{(1),(1)}^{(1)}$, we have

$$
\left|\widetilde{M}_{\tilde{\pi}, \tilde{\sigma}}^{\bullet ; \tilde{\lambda}}\right|=\left|\widetilde{M}_{\pi, \sigma}^{\bullet ; \lambda}\right| .
$$

Indeed, from any $\lambda$-list of maps $M \in \widetilde{M}_{\pi, \sigma}^{\bullet ; \lambda}$ we can canonically create a $\tilde{\lambda}$-list of map $\widetilde{M} \in \widetilde{M}_{\tilde{\pi}, \tilde{\sigma}}^{\bullet ; \tilde{\lambda}}$ by erasing the last $|\lambda|-|\tilde{\lambda}|$ components. This procedure is reversible, since we can add new $M_{1}$ components to $\widetilde{M}$. Then we have

$$
\left|\widetilde{M}_{\pi, \sigma}^{\bullet ; \lambda}\right|=\sum_{\nu: \nu \preceq \lambda}\left|\widetilde{M}_{\pi, \sigma}^{\nu ; \lambda}\right| \stackrel{\text { Observation } 3.12}{=} \sum_{\nu: \nu \preceq \lambda} \frac{z_{\lambda}}{z_{\nu}}\left|\widetilde{\mathcal{G}}_{\pi, \sigma}^{\nu ; \lambda}\right| .
$$

Hence

$$
\left[\beta^{d(\pi, \sigma ; \lambda)}\right] c_{\pi, \sigma}^{\lambda}=\sum_{\nu: \nu \preceq \lambda} \frac{z_{\lambda}}{z_{\nu}}\left|\widetilde{\mathcal{G}}_{\pi, \sigma}^{\nu ; \lambda}\right|
$$

From Lemma 5.12 we conclude that the leading coefficient of $c_{\pi, \sigma}^{\lambda}$ coincides with the leading coefficient of the polynomial

$$
\left(G_{\eta}\right)_{\pi, \sigma}^{\lambda ; \lambda}:=\sum_{\delta \in \mathcal{G}_{\pi, \sigma}^{\lambda ; \lambda}} \beta^{\operatorname{stat}(\delta)}
$$

see (14), and that both are of the same degree. As we shall see, Lemma 5.12 provides the second expression for the leading coefficient of the polynomial $c_{\pi, \sigma}^{\lambda}$.

\section{Acknowledgements}

The author is greatly thankful to Piotr Śniady for valuable and fruitful discussions, which greatly improved this text. 


\section{A Top-degree parts in the Matchings-Jack Conjecture and the $b$-Conjecture}

We shall prove that our result about the top-degree part in the Matchings-Jack Conjecture presented in Theorem 1.5 and the result of Dołęga [Doł17, Theorem 1.5] about the topdegree part in $b$-Conjecture are equivalent.

First note that the polynomials $c_{\pi, \sigma}^{\lambda}$ and $h_{\pi, \sigma}^{\lambda}$ are related as follows

$$
\begin{aligned}
\sum_{n \geqslant 1} t^{n} \sum_{\lambda, \pi, \sigma \vdash n} h_{\pi, \sigma}^{\lambda} p_{\pi}(\mathbf{x}) p_{\sigma}(\mathbf{y}) p_{\lambda}(\mathbf{z}) & = \\
& \alpha t \frac{\partial}{\partial t} \log \left(\sum_{n \geqslant 0} t^{n} \sum_{\lambda, \pi, \sigma \vdash n} \frac{c_{\pi, \sigma}^{\lambda}}{\alpha^{\ell(\lambda)} z_{\lambda}} p_{\pi}(\mathbf{x}) p_{\sigma}(\mathbf{y}) p_{\lambda}(\mathbf{z})\right)
\end{aligned}
$$

and

$$
\begin{aligned}
\sum_{n \geqslant 0} t^{n} \sum_{\lambda, \pi, \sigma \vdash n} \frac{c_{\pi, \sigma}^{\lambda}}{\alpha^{\ell(\lambda)} z_{\lambda}} p_{\pi}(\mathbf{x}) p_{\sigma}(\mathbf{y}) p_{\lambda}(\mathbf{z}) & = \\
& \exp \left(\sum_{n \geqslant 1} \frac{1}{\alpha n} t^{n} \sum_{\lambda, \pi, \sigma \vdash n} h_{\pi, \sigma}^{\lambda} p_{\pi}(\mathbf{x}) p_{\sigma}(\mathbf{y}) p_{\lambda}(\mathbf{z})\right),
\end{aligned}
$$

see (1) and (2). Multiplication of power-sum symmetric functions expresses as follows $p_{\lambda_{1}}(\mathbf{z}) \cdot p_{\lambda_{1}}(\mathbf{z})=p_{\lambda_{1} \cup \lambda_{1}}(\mathbf{z})$ in the terms of concatenations of relevant partitions. Notice that for $\lambda=(n)$ only the linear term in the expansion of the logarithm on the RHS of (16) contribute to the coefficient of $p_{\lambda}(\mathbf{z})$.

In Theorem 1.5 we showed that the leading coefficient of $c_{\pi, \sigma}^{\lambda}$ can be expressed in the following way:

$$
\left[\beta^{d(\pi, \sigma ; \lambda)}\right] c_{\pi, \sigma}^{\lambda}=\mid M \in M_{\pi, \sigma}^{\lambda ; \lambda}: M \text { is unhandled } \mid
$$

where $M_{\pi, \sigma}^{\lambda ; \lambda}$ is the set of $\lambda$-lists of unicellular maps with the white and black vertices distribution given by $\pi$ and $\sigma$ respectively.

On the other hand, Dołęga [Doł17, Theorem 1.5] showed that the leading coefficient of $h_{\pi, \sigma}^{(n)}$ can be expressed in the following way:

$$
\left[\beta^{d(\pi, \sigma ;(n))}\right] h_{\pi, \sigma}^{(n)}=\mid M \in M_{\pi, \sigma}^{(n)}: M \text { is unhandled } \mid
$$

where $M_{\pi, \sigma}^{(n)}$ is the set of unicellular maps with the white and black vertices distribution given by $\pi$ and $\sigma$ respectively.

We investigate the $\left[p_{(n)}(\mathbf{z})\right]$ coefficient in both sides of (16). We have

$$
\begin{aligned}
& t^{n} \sum_{\pi, \sigma \vdash n} h_{\pi, \sigma}^{(n)} p_{\pi}(\mathbf{x}) p_{\sigma}(\mathbf{y})=\alpha t \frac{\partial}{\partial t}\left(t^{n} \sum_{\pi, \sigma \vdash n} \frac{c_{\pi, \sigma}^{(n)}}{\alpha z_{(n)}} p_{\pi}(\mathbf{x}) p_{\sigma}(\mathbf{y})\right)= \\
& \alpha n t^{n} \sum_{\pi, \sigma \vdash n} \frac{c_{\pi, \sigma}^{(n)}}{\alpha n} p_{\pi}(\mathbf{x}) p_{\sigma}(\mathbf{y})
\end{aligned}
$$


hence $c_{\pi, \sigma}^{(n)}$ and $h_{\pi, \sigma}^{(n)}$ are equal.

Since $c_{\pi, \sigma}^{(n)}=h_{\pi, \sigma}^{(n)}$, it might seem that our result extends the result of Dołega. However, a more subtle analysis of relationships between the coefficients of $c_{\pi, \sigma}^{\lambda}$ and $h_{\pi, \sigma}^{\lambda}$ shows that both results are equivalent.

The power series expansion of the exponential function in (17) gives us

$$
\begin{aligned}
& \sum_{n \geqslant 0} t^{n} \sum_{\lambda, \pi, \sigma \vdash n} \frac{c_{\pi, \sigma}^{\lambda}}{\alpha^{\ell(\lambda)} z_{\lambda}} p_{\pi}(\mathbf{x}) p_{\sigma}(\mathbf{y}) p_{\lambda}(\mathbf{z})= \\
& \sum_{k \geqslant 0} \frac{1}{k !}\left(\sum_{s \geqslant 1} \frac{1}{s} t^{s} \sum_{\lambda, \pi, \sigma \vdash s} \frac{h_{\pi, \sigma}^{\lambda}}{\alpha} p_{\pi}(\mathbf{x}) p_{\sigma}(\mathbf{y}) p_{\lambda}(\mathbf{z})\right)^{k} .
\end{aligned}
$$

We denote by $\mathcal{P}_{k}^{\lambda, \pi, \sigma}$ the set of triplets of lists of partitions

$$
\left(\left(\lambda^{1}, \ldots, \lambda^{k}\right),\left(\pi^{1}, \ldots, \pi^{k}\right),\left(\sigma^{1}, \ldots, \sigma^{k}\right)\right)
$$

such that

$$
\bigcup_{i=1}^{k} \lambda^{i}=\lambda, \quad \bigcup_{i=1}^{k} \mu^{i}=\mu, \quad \bigcup_{i=1}^{k} \sigma^{i}=\sigma
$$

and for each $i$ we have $\left|\lambda^{i}\right|=\left|\pi^{i}\right|=\left|\sigma^{i}\right|$.

Let us investigate the $\left[p_{\pi}(\mathbf{x}) p_{\sigma}(\mathbf{y}) p_{\lambda}(\mathbf{z})\right]$ coefficient in both sides of (18). We have

$$
t^{n} \frac{c_{\pi, \sigma}^{\lambda}}{\alpha^{\ell(\lambda)} z_{\lambda}}=t^{n} \sum_{1 \leqslant k \leqslant \ell(\lambda)} \frac{1}{k !} \sum_{\substack{\left.\left(\lambda^{1}, \ldots, \lambda^{k}\right),\left(\pi^{1}, \ldots, \pi^{k}\right),\left(\sigma^{1}, \ldots, \sigma^{k}\right)\right) \in \mathcal{P}_{k}^{\lambda, \pi, \sigma}}} \prod_{i=1}^{k} \frac{1}{\left|\lambda^{i}\right|} \frac{h_{\pi^{i}, \sigma^{i}}^{\lambda^{i}}}{\alpha} .
$$

Dołęga and Féray [DF17, Theorem 1.2] gave the following bound on the degree

$$
\operatorname{deg} h_{\pi^{i}, \sigma^{i}}^{\lambda^{i}} \leqslant\left|\lambda^{i}\right|+2-\ell\left(\lambda^{1}\right)-\ell\left(\pi^{i}\right)-\ell\left(\sigma^{i}\right) .
$$

Hence, each summand of the first sum on the right-hand side of (19) has degree equal to at most

$$
n+2 k-\ell(\lambda)-\ell(\pi)-\ell(\sigma),
$$

and the maximal bound may be achieved only for summands corresponding to $k=\ell(\lambda)$. For such a summand, its bound on the degree is the same as the bound on the degree for the left-hand side of (19) given by (3). We have

$$
\frac{1}{z_{\lambda}}\left[\alpha^{d(\pi, \sigma ; \lambda)}\right] c_{\pi, \sigma}^{\lambda}=\frac{1}{\ell(\lambda) !} \sum_{\substack{\left.\left(\lambda^{1}, \ldots, \lambda^{\ell(\lambda)}\right),\left(\pi^{1}, \ldots, \pi^{\ell(\lambda)}\right),\left(\sigma^{1}, \ldots, \sigma^{\ell(\lambda)}\right)\right) \in \mathcal{P}_{\ell(\lambda)}^{\lambda, \pi, \sigma}}}^{\ell(\lambda)} \prod_{i=1}^{\left|\lambda^{i}\right|}\left[\alpha^{\left|\lambda_{i}\right|+1-\ell(\pi)-\ell(\sigma)}\right] h_{\pi^{i}, \sigma^{i}}^{\lambda_{i}} .
$$


For a Young diagram $\lambda=\left(\lambda_{1}, \ldots, \lambda_{k}\right)$, denote by $\mathcal{C}_{\lambda}$ the set of all compositions of a type $\lambda$, i.e. the set of all lists $\left(\lambda_{\sigma(1)}, \ldots, \lambda_{\sigma(k)}\right)$, for some $\sigma \in \mathfrak{S}(n)$. Observe that

$$
\left|\mathcal{C}_{\lambda}\right|=\frac{\ell(\lambda) !}{\sum_{i} m_{i}(\lambda) !}
$$

Observe that for $k=\ell(\lambda)$ the first list in any triplet from $\mathcal{P}_{k}^{\lambda, \pi, \sigma}$ is a composition of a type of the Young diagram $\lambda$. We have

$$
\begin{aligned}
\frac{1}{z_{\lambda}}\left[\alpha^{d(\pi, \sigma ; \lambda)}\right] c_{\pi, \sigma}^{\lambda}=\frac{1}{\ell(\lambda) !}\left|\mathcal{C}_{\lambda}\right| & \sum \prod_{i=1}^{\ell(\lambda)} \frac{1}{\lambda_{i}}\left[\alpha^{\lambda_{i}+1-\ell(\pi)-\ell(\sigma)}\right] h_{\pi^{i}, \sigma^{i}}^{\left(\lambda_{i}\right)} \\
& \left(\left(\left(\lambda_{1}\right), \ldots,\left(\lambda_{\ell(\lambda)}\right)\right),\right. \\
& \left(\pi^{1}, \ldots, \pi^{\ell(\lambda)}\right), \\
& \left.\left(\sigma^{1}, \ldots, \sigma^{\ell(\lambda)}\right)\right) \in \mathcal{P}_{\ell(\lambda)}^{\lambda, \pi, \sigma}
\end{aligned}
$$

and hence

$$
\begin{aligned}
{\left[\alpha^{d(\pi, \sigma ; \lambda)}\right] c_{\pi, \sigma}^{\lambda}=} & \sum \prod_{i=1}^{\ell(\lambda)}\left[\alpha^{\lambda_{i}+1-\ell(\pi)-\ell(\sigma)}\right] h_{\pi^{i}, \sigma^{i}}^{\left(\lambda_{i}\right)} \\
& \left(\left(\left(\lambda_{1}\right), \ldots,\left(\lambda_{\ell(\lambda)}\right)\right),\right. \\
& \left(\pi^{1}, \ldots, \pi^{\ell(\lambda)}\right), \\
& \left.\left(\sigma^{1}, \ldots, \sigma^{\ell(\lambda)}\right)\right) \in \mathcal{P}_{\ell(\lambda)}^{\lambda, \pi, \sigma}
\end{aligned}
$$

Dołega's result [Doł17, Theorem 1.5] shows us that

$$
\left[\alpha^{\lambda_{i}+1-\ell(\pi)-\ell(\sigma)}\right] h_{\pi^{i}, \sigma^{i}}^{\left(\lambda_{i}\right)}=\mid M \in M_{\pi^{i}, \sigma^{i}}^{\left(\lambda_{i}\right)}: M \text { is unhandled } \mid .
$$

Directly from the definition of $\mathcal{P}_{\ell(\lambda)}^{\lambda, \pi, \sigma}$ we obtain that

$$
\left[\alpha^{d(\pi, \sigma ; \lambda)}\right] c_{\pi, \sigma}^{\lambda}=\mid M \in M_{\pi, \sigma}^{\lambda ; \lambda}: M \text { is unhandled } \mid
$$

which allows us to conclude the equivalence of both results.

\section{References}

[AF17] Per Alexandersson and Valentin Féray. Shifted symmetric functions and multirectangular coordinates of Young diagrams. J. Algebra, 483:262-305, 2017.

[DF16] Maciej Dołęga and Valentin Féray. Gaussian fluctuations of Young diagrams and structure constants of Jack characters. Duke Math. J., 165(7):1193-1282, 2016. 
[DF17] Maciej Dołęga and Valentin Féray. Cumulants of jack symmetric functions and the b-conjecture. Transactions of the American Mathematical Society, 369(12):9015-9039, 2017.

[DH92] Persi Diaconis and Phil Hanlon. Eigen-analysis for some examples of the Metropolis algorithm. In Hypergeometric functions on domains of positivity, Jack polynomials, and applications (Tampa, FL, 1991), volume 138 of Contemp. Math., pages 99-117. Amer. Math. Soc., Providence, RI, 1992.

[Doł17] Maciej Dołęga. Top degree part in $b$-conjecture for unicellular bipartite maps. Electron. J. Combin., 24(3):\#P3.24, 2017.

[Fér12] Valentin Féray. On complete functions in Jucys-Murphy elements. Ann. Comb., 16(4):677-707, 2012.

[GJ96] Ian P. Goulden and David M. Jackson. Connection coefficients, matchings, maps and combinatorial conjectures for Jack symmetric functions. Trans. Amer. Math. Soc., 348(3):873-892, 1996.

[HSS92] Philip J. Hanlon, Richard P. Stanley, and John R. Stembridge. Some combinatorial aspects of the spectra of normally distributed random matrices. In Hypergeometric functions on domains of positivity, Jack polynomials, and applications (Tampa, FL, 1991), volume 138 of Contemp. Math., pages 151-174. Amer. Math. Soc., Providence, RI, 1992.

[IK99] Vladimir Ivanov and Sergei Kerov. The algebra of conjugacy classes in symmetric groups, and partial permutations. Teor. Predst. Din. Sist. Komb. $i$ Algoritm. Metody., 256(3):95-120, 265, 1999.

[Jac71] Henry Jack. I. -a class of symmetric polynomials with a parameter. Proceedings of the Royal Society of Edinburgh. Section A. Mathematical and Physical Sciences, 69, 01 1970/1971.

[Kan93] Jyoichi Kaneko. Selberg integrals and hypergeometric functions associated with Jack polynomials. SIAM J. Math. Anal., 24(4):1086-1110, 1993.

[Ker00] Sergei Kerov. Anisotropic Young diagrams and symmetric Jack functions. Funktsional. Anal. i Prilozhen., 34(1):51-64, 96, 2000.

[KO94] Sergei Kerov and Grigori Olshanski. Polynomial functions on the set of Young diagrams. C. R. Acad. Sci. Paris Sér. I Math., 319(2):121-126, 1994.

[KS96] Friedrich Knop and Siddhartha Sahi. Difference equations and symmetric polynomials defined by their zeros. Internat. Math. Res. Notices, pages 473486, 1996.

[KV16] Andrei L. Kanunnikov and Ekaterina A. Vassilieva. On the matchings-Jack conjecture for Jack connection coefficients indexed by two single part partitions. Electron. J. Combin., 23(1):\#P1.53, 2016.

[KVP17] Andrei L. Kanunnikov, Ekaterina A. Vassilieva, and Valentin V. Promyslov. On the matchings-Jack and hypermap-Jack conjectures for labelled matchings and star hypermaps. arXiv:1712.08246, 2017. 
[La 09] Michael Andrew La Croix. The combinatorics of the Jack parameter and the genus series for topological maps. PhD thesis, University of Waterloo, 2009.

[Las08] Michel Lassalle. A positivity conjecture for Jack polynomials. Math. Res. Lett., 15(4):661-681, 2008.

[Las09] Michel Lassalle. Jack polynomials and free cumulants. Adv. Math., 222(6):2227-2269, 2009.

[LZ04] Sergei K. Lando and Alexander K. Zvonkin. Graphs on surfaces and their applications, volume 141 of Encyclopaedia of Mathematical Sciences. SpringerVerlag, Berlin, 2004. With an appendix by Don B. Zagier, Low-Dimensional Topology, II.

[Mac15] Ian G. Macdonald. Symmetric functions and Hall polynomials. Oxford Classic Texts in the Physical Sciences. The Clarendon Press, Oxford University Press, New York, second edition, 2015. With contribution by A. V. Zelevinsky and a foreword by Richard Stanley, Reprint of the 2008 paperback edition.

[Nak96] Hiraku Nakajima. Jack polynomials and Hilbert schemes of points on surfaces. arXiv:alg-geom/9610021, 1996.

[Oko03] Andrei Okounkov. The uses of random partitions. In Fourteenth International Congress on Mathematical Physics, pages 379-403, 2003.

[OO97] Andrei Okounkov and Grigori Olshanski. Shifted Jack polynomials, binomial formula, and applications. Math. Res. Lett., 4(1):69-78, 1997.

[Sta89] Richard P. Stanley. Some combinatorial properties of Jack symmetric functions. Adv. Math., 77(1):76-115, 1989.

[Śn15] Piotr Śniady. Top degree of Jack characters and enumeration of maps. arXiv:1506.06361v2, 2015.

[Śn19] Piotr Śniady. Asymptotics of jack characters. Journal of Combinatorial Theory, Series A, 166:91-143, Aug 2019. 